\title{
Integral field observations of the blue compact galaxy Haro14 ${ }^{\star}$ Star formation and feedback in dwarf galaxies
}

\author{
L. M. Cairós ${ }^{1}$ and J. N. González-Pérez ${ }^{2}$ \\ ${ }^{1}$ Institut für Astrophysik, Georg-August-Universität, Friedrich-Hund-Platz 1, 37077 Göttingen, Germany \\ e-mail: luzma@astro.physik.uni-goettingen.de \\ 2 Hamburger Sternwarte, Gojenbergsweg 112, 21029 Hamburg, Germany \\ e-mail: jgonzalezperez@hs.uni-hamburg.de
}

Received 10 September 2016 / Accepted 21 December 2016

\begin{abstract}
Context. Low-luminosity, gas-rich blue compact galaxies (BCG) are ideal laboratories to investigate the triggering and propagation of star formation in galaxies, the effects of massive stellar feedback within a shallow gravitational potential, and the enrichment of the interstellar medium.

Aims. We aim to probe the morphology, stellar content, and kinematics, along with the nebular excitation and ionization mechanism, in the BCG Haro 14 by means of integral field observations.

Methods. We observed Haro 14 at the Very Large Telescope, working with the Visible Multi-Object Spectrograph. From these data we build maps in continuum and in the brighter emission lines, produce line-ratio maps (interstellar extinction, density, and diagnosticline ratios), and obtain the velocity and velocity dispersion fields. We also generate the integrated spectrum of the major $\mathrm{H}$ II regions and young stellar clusters identified in the maps to determine reliable physical parameters and oxygen abundances.

Results. We find as follows: i) the current star formation in Haro 14 is spatially extended with the major H II regions placed along a linear (chain-like) structure, elongated in the north-south direction, and in a horseshoe-like curvilinear feature that extends about $760 \mathrm{pc}$ eastward; the continuum emission is more concentrated and peaks close to the galaxy center; ii) two different episodes of star formation are present in the central galaxy regions: the recent starburst, with ages $\leq 6 \mathrm{Myr}$ and the intermediate-age clusters, with ages between 10 and $30 \mathrm{Myr}$; these stellar components rest on a several Gyr old underlying host galaxy; iii) the $\mathrm{H} \alpha / \mathrm{H} \beta$ pattern is inhomogeneous, with excess color values varying from $E(B-V)=0.04$ up to $E(B-V)=1.09$; iv) shocks play a significant role in the galaxy; and v) the velocity field displays a complicated pattern with regions of material moving toward us in the east and north galaxy areas.

Conclusions. The morphology of Haro 14, its irregular velocity field, and the presence of shocks speak in favor of a scenario of triggered star formation. Ages of the knots, i.e., $6 \mathrm{Myr}$ for the starburst and 10-30 Myr for the central clusters, are consistent with the ongoing burst being triggered by the collective action of stellar winds and supernovae originated in the central clusters.
\end{abstract}

Key words. galaxies: abundances - galaxies: individual: Haro14 - galaxies: dwarf - galaxies: ISM - galaxies: star formation galaxies: stellar content

\section{Introduction}

Dwarf galaxies have become very popular objects in contemporary astronomy, and deservedly so because these small and elusive systems play an essential role in the understanding of various fundamental astrophysical problems. Dwarf galaxies constitute an unparalleled link to the early Universe since within the framework of a cold dark matter Universe, galaxies form by hierarchical merger of smaller objects (Springel et al. 2006). As building blocks of more massive systems, dwarfs provide key clues in the processes involved in galaxy assembly and evolution.

Among dwarfs, the low luminosity $\left(M_{B} \geq-18\right)$ and low metallicity $\left(1 / 50 Z_{\odot} \leq Z \leq 1 / 2 Z_{\odot}\right.$; Kunth \& Östlin 2000) blue compact galaxies (BCG) appear as ideal laboratories to probe the still poor understood process of star formation (SF) at large scales. They are gas-rich galaxies, experiencing intense ongoing

\footnotetext{
* Based on observations made with ESO Telescopes at Paranal Observatory under program ID 079.B-0445.
}

SF. Because they are small and structurally simple, BCGs can neither sustain density waves nor suffer from disk instabilities and, therefore, offer a unique chance to investigate the SF in an uncomplicated environment.

The study of the effects of energy feedback from massive stars into the surrounding medium in BCGs is also crucial. Due to their shallow potentials, it has been suggested that dwarf systems can actually be losing mass (Dekel \& Silk 1986). If this were the case, this would have important consequences for the picture of galaxy formation and evolution, process of SF, and enrichment of the intergalactic medium (IGM). Furthermore, understanding the feedback process will help to establish the role of star-forming galaxies in the reioinization of the IGM in the early Universe (Grimes et al. 2007).

Motivated by the importance of these issues we initiated a project focused on the investigation of BCGs and, in particular, on their ongoing SF episode and the impact of their massive, young stars on the surrounding interstellar medium (ISM). To this end we undertook a comprehensive analysis of a sample of 
about 40 objects by means of integral field spectroscopy (IFS). Integral field unit (IFU) instruments are particularly suited to probe small, highly asymmetric and compact systems such as BCGs because in just one shot they provide spectrophotometric and kinematic information on a large portion of the galaxy; generally the whole starburst is covered. A detailed description of the project as well as our first results can be found in Cairós et al. (2009b,a, 2010, 2012, 2015).

The outcomes of this work posed some puzzling new questions and raised the need for further analyses; in particular, the results found for several galaxies warrant additional work. While analyses of large datasets are fundamental to attack a new field of research and a prerequisite to devise new ideas and theories, the further confrontation with the individual characteristics of particular objects is the appropriate way to validate the conclusions.

In this paper we present a detailed analysis of the galaxy Haro 14. This is a nearby dwarf system $\left(M_{B}=-16.92\right)$, which appears classified as a BCG after its inclusion in the pioneering work by Thuan \& Martin (1981). Broadband optical and near-infrared (NIR) data have been presented in Marlowe et al. (1997), Doublier et al. (1999), Gil de Paz et al. (2003), Gil de Paz \& Madore (2005), Hunter \& Elmegreen (2006), Doublier et al. (2001) and Noeske et al. (2003). Optical integrated spectrophotometry has been carried out by Moustakas \& Kennicutt (2006) and Hunter \& Hoffman (1999). We observed Haro 14, together with seven other BCGs, working with the VIsible Multi-Object Spectrograph (VIMOS; Le Fèvre et al. 2003). First results for the whole sample, including emission and diagnostic-line maps, interstellar extinction and electron density maps, and velocity and velocity dispersion fields, were presented in Cairós et al. (2015). Our analysis revealed Haro 14 as a very intriguing object. Its morphology, with the continuum emission concentrated in the central galaxy regions and the current SF activity taking place in several knots distributed across the whole observed field, suggests the presence of different episodes of SF. We identify a bubble-like curvilinear feature, extending about $760 \mathrm{pc}$ from the continuum peak, and measure high values of [S II] $\lambda \lambda 6717,6731 / \mathrm{H} \alpha$ in the galaxy outskirts, signature of the presence of shocks. These findings motivated further work on this object, whose results are presented in this paper.

\section{The data}

\subsection{Observations and data process}

Spectrophotometric observations of Haro 14 were performed at the Very Large Telescope (VLT; ESO Paranal Observatory, Chile), with VIMOS in its IFU mode. They were carried out in visitor mode, during the nights of August 19-20, 2007. The blue (HR-Blue; 4150-6200 $\AA$ ) and orange (HR-Orange; 5250-7400 $\AA$ ) grisms in high resolution mode (dispersions of $0.51 \AA \mathrm{pix}^{-1}$, and of $0.60 \AA \mathrm{pix}^{-1}$, respectively) were used. A field of view (FOV) of $27^{\prime \prime} \times 27^{\prime \prime}$ on the sky was mapped with an spatial sampling of 0".67 (see Fig. 1 and Table 1).

Haro 14 was observed for $4320 \mathrm{~s}$ with both the HR-Blue and HR-Orange grisms. The weather conditions were good, the seeing was in the range $0.94-1.53$ arcsec, and exposures were taken at airmass 1.03-1.23. The spectrophotometric standard EG 274 was observed for flux calibration.

The data were processed using the ESO VIMOS pipeline (version 2.1.11) via the graphical user interface GASGANO. A complete description of observations and the data analysis has been presented in Cairós et al. (2015).

A125, page 2 of 16

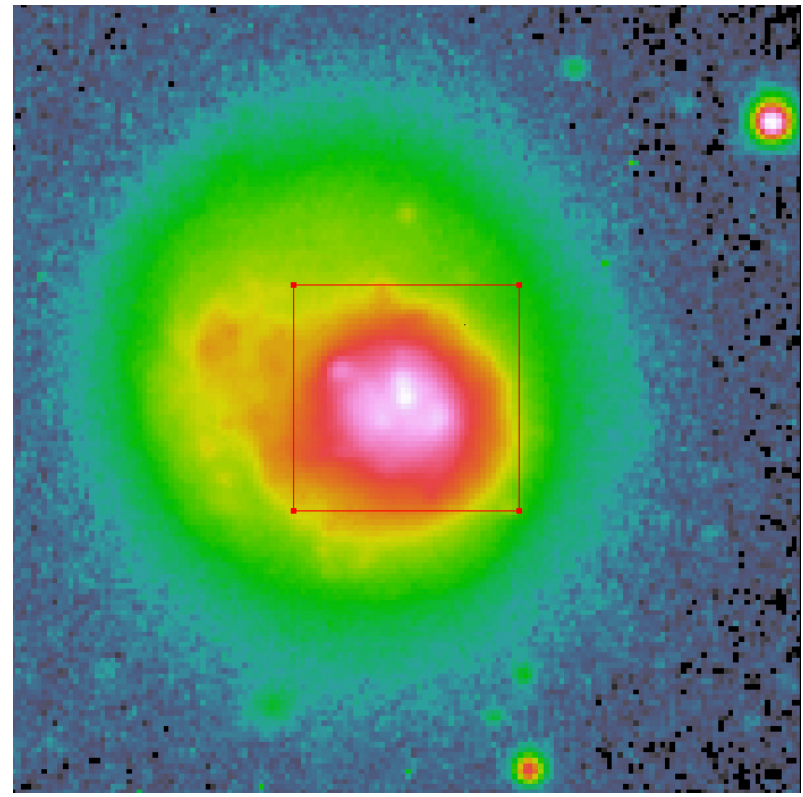

Fig. 1. $V$-band image of Haro 14 retrieved from NED and published in Hunter \& Elmegreen (2006); the field of view is $1.5^{\prime}$ and the central box indicates the $27^{\prime \prime} \times 27^{\prime \prime}$ covered by VIMOS. North is up and east to the left (also in all maps shown from here on).

Table 1. Basic parameters for Haro 14 and collection of data from the literature.

\begin{tabular}{lcc}
\hline \hline Parameter & Data & Reference \\
\hline Other names & $\begin{array}{c}\text { NGC 0244, UGCA 10, } \\
\text { VV 728, PGC 2675 }\end{array}$ \\
RA (J2000) & $00^{\mathrm{h}} 45^{\mathrm{m}} 46^{\mathrm{s}} 4$ \\
Dec (J2000) & $-15^{\circ} 35^{\prime} 49^{\prime \prime}$ & \\
$V_{\text {heliocentric }}$ & $941 \pm 0.06 \mathrm{~km} \mathrm{~s}^{-1}$ & \\
Distance & $13.0 \pm 0.09 \mathrm{Mpc}$ & \\
$D_{25}$ & $1.09 \pm 0.06$ arcmin & RC3 \\
$A_{B}$ & 0.075 & \\
Morphology & SOpec; BCG & RC3; TM81 \\
\hline$m_{B}$ & $13.65 \pm 0.05^{a}$ & GP03 \\
$m_{R}$ & $12.91 \pm 0.14^{a}$ & GP03 \\
$M_{B}$ & $-16.92^{b}$ & \\
$M_{\mathrm{HI}}$ & $3.2 \times 10^{8} M_{\odot}$ & TM81 \\
$M_{T}$ & $3.8 \times 10^{8} M_{\odot}$ & TM81 \\
\hline
\end{tabular}

Notes. RA, Dec, heliocentric velocity, distance, apparent major isophotal diameter $D_{25}$ measured at a surface brightness level of $25.0 \mathrm{mag} \operatorname{arcsec}^{-2}$, and Galactic extinction are taken from NED. ${ }^{1}$ The distance was calculated using a Hubble constant of $73 \mathrm{~km} \mathrm{~s}^{-1} \mathrm{Mpc}^{-1}$, and taking into account the influence of the Virgo cluster, the Great Attractor, and the Shapley supercluster. (a) Integrated magnitudes from Gil de Paz et al. (2003), corrected for Galactic extinction; (b) absolute magnitude in the $B$ band computed from the tabulated $B$ magnitude and distance.

References. RC3: de Vaucouleurs et al. (1991); TM81: Thuan \& Martin (1981); and GP03: Gil de Paz et al. (2003).

\subsection{Creating the $2 D$ maps}

The relevant parameters of the emission lines (line flux, centroid position, line width, and continuum) were measured by fitting single Gaussian line profiles. The fit was carried out with

1 http://nedwww.ipac.caltech.edu/ 
the Trust-region algorithm for non-linear least squares, using the function fit of MATLAB; reasonable initial values and lower and upper bounds for the parameters were provided. We run an automatic procedure, which fits a series of lines for every spaxel, namely, $\mathrm{H} \beta$, [O III] $\lambda 4959$, [O III] $\lambda 5007$, [O I] $] 6300, \mathrm{H} \alpha$, $[\mathrm{N}$ II $] \lambda 6583$, and [S II] $\lambda 6716$, [S II] 26731 . Errors of the parameters are provided by the fitting code.

Computing the fluxes of the Balmer lines in emission is not a straightforward task, as these fluxes can be considerably affected by the absorption of the underlying population of stars. To take this effect into account in the $\mathrm{H} \beta$ profile, in which absorption wings are clearly visible, we simply fit the line profile with two Gaussian functions (one in emission and one in absorption) deriving in this way absorption and emission fluxes simultaneously. In the case of $\mathrm{H} \alpha$ the absence of visible absorption wings makes a reliable decomposition impossible, and an alternative approach must be adopted. We consider the equivalent width in absorption in $\mathrm{H} \alpha$ be equal than in $\mathrm{H} \beta$. Assuming the same absorption equivalent width for the Balmer lines, when the values cannot be fitted individually, has been traditionally the most common strategy adopted (McCall et al. 1985; Popescu \& Hopp 2000). It is a supposition that is well founded by the predictions of evolutionary synthesis models (Olofsson 1995).

In low-surface-brightness (LSB) regions, a spatial smoothing procedure was applied to increase the accuracy of the fits. Depending on the signal-to-noise ratio $(\mathrm{S} / \mathrm{N})$ of the spaxel, the closest 5,9 , or 13 spaxels were averaged before the fit was carried out. In this way, we maintain the spatial resolution of the bright regions of the galaxy, while obtaining a reasonable $\mathrm{S} / \mathrm{N}$ of the faint parts, but with a lower spatial resolution.

The parameters of the fit were used subsequently to construct the 2D maps presented here, taking advantage of the fact that the combined VIMOS data are arranged in a regular $44 \times 44$ matrix. Only spaxels with fluxes higher than $3 \sigma$ level are considered.

Continuum maps at different spectral ranges were obtained by summing the flux within specific wavelength intervals, selected so as to avoid strong emission lines or residuals from the sky spectrum subtraction. Also an integrated continuum map was produced by summing over the whole spectral range, but masking the spectral regions with a significant contribution of emission lines.

Line ratios maps for lines falling in the wavelength range of either grism were simply derived by dividing the corresponding flux maps. Line ratio maps include again only those spaxels containing values higher than the $3 \sigma$ level.

In the case of the $\mathrm{H} \alpha / \mathrm{H} \beta$, the line ratio map was derived after registering and shifting the $\mathrm{H} \alpha$ map to spatially match the $\mathrm{H} \beta$ map. The amount of shift was calculated using the difference in position of the center of the brighter HII-regions. The shifting (about $0.27^{\prime \prime}$ and $0.20^{\prime \prime}$ in RA and Dec, respectively) was applied using a bilinear interpolation. In order to correct for the fact that $\mathrm{H} \alpha$ and $\mathrm{H} \beta$ had been observed under different seeing conditions, the $\mathrm{H} \alpha$ map has been convolved with a Gaussian profile, to match both point spread functions.

All the maps present in this work were corrected for interstellar extinction in terms of spaxels. The method is described in Sect. 3.5.2 for integrated spectra; in the maps, the same method was applied to every spaxel.

\section{Results}

Haro 14 is made of an extended high-surface-brightness (HSB) star-forming region, placed atop a smooth LSB underlying stellar component (Fig. 1); this redder population has a diameter of about $4.12 \mathrm{kpc}$ (de Vaucouleurs et al. 1991), whereas the central HSB region, which has been resolved in several H II-regions and/or stellar clusters (Doublier et al. 1999; Noeske et al. 2003; Cairós et al. 2015), fills the VIMOS FOV (about $1.7 \times 1.7 \mathrm{kpc}$ ). According to the morphological classification made by Loose \& Thuan (1986), the galaxy belongs to the most common BCG type, namely, the iE objects, which represent about $70 \%$ of the $\mathrm{BCG}$ population.

\subsection{Stellar and ionized gas morphology}

Continuum and emission-line intensity maps of Haro 14, derived from the VIMOS data as described in Sect. 2.2, are shown in Fig. 2. At the galaxy distance, the resolution element (spaxel) translates into $42 \mathrm{pc}$.

The continuum registers photons produced in the photosphere of the stars and, hence, traces the stellar component of the galaxy. On the other hand, hydrogen recombination-line maps capture photons produced by the excited atoms of the gas, as a recombined electron cascades down to the ground state. Forbidden lines of trace species, such as [O III] or [N II], the other main type of emission-lines detected in the spectra of BCGs/H IIgalaxies, are also generated in the ionized interstellar gas; these lines result from the emission of photons from collisionally excited levels. Therefore, emission-line maps trace regions of photoionized hydrogen, i.e., regions that are rich in hot OB stars.

Substantial deviations between the stellar and ionized gas distributions in Haro 14 are evident from Fig. 2. In the continuum, the intensity reaches its maxima close to the galaxy center, where an ensemble of central stellar clusters is identified, encircled by a roughly regular stellar envelope. The two major clusters were labeled as A, B following Noeske et al. (2003). The maximum of emission in the continuum, which does not depend on the spectral window selected, is cospatial with Knot A.

The maps in warm ionized gas exhibit a quite different morphology. Here, the peaks of intensity do not gather in the central galaxy regions, but they are scattered across the whole mapped area. A linear (chain-like) structure extends in the north-south direction, and a horseshoe-like curvilinear feature departs from these central knots and extends about 12'.0 (760 pc) eastward; the morphology and size of this curvilinear feature suggest the presence of a large-scale expanding structure (supershell or superbubble; Tenorio-Tagle \& Bodenheimer 1988). The major SF knots are labeled in Fig. 3.

The central galaxy region displays essentially the same pattern in all the emission-line maps with the intensity maximum always situated in Knot 2 (see Cairós et al. 2015, for a complete collection of emission-line maps for Haro 14). Extended diffuse gas emission surrounds these major H II regions. Several fainter lumps emerge around the central SF major area: the largest lump at the west (Knot 8 in Fig. 3) and some others detectable in the southeast. These blobs are better seen in the higher excitation [O III] line, where they are well visible as condensations separated from the galaxy main body, whereas they are almost undetectable in the [S II] lower ionization line (see Fig. 2).

None of the bright continuum peaks (Knots A and B) spatially coincide with a peak in the ionized-gas distribution, indicating that the HSB clusters are dominated by non-ionizing stars (ages larger than $10 \mathrm{Myr}$ ). The continuum peak (Knot A) is located about 168 pc south of the emission-line peak (Knot 2).

In order to understand the star-forming history of a galaxy, its different stellar components must be first distinguished and characterized. By comparing the emission-line and continuum morphology in Haro 14, we get the first insights on its stellar 

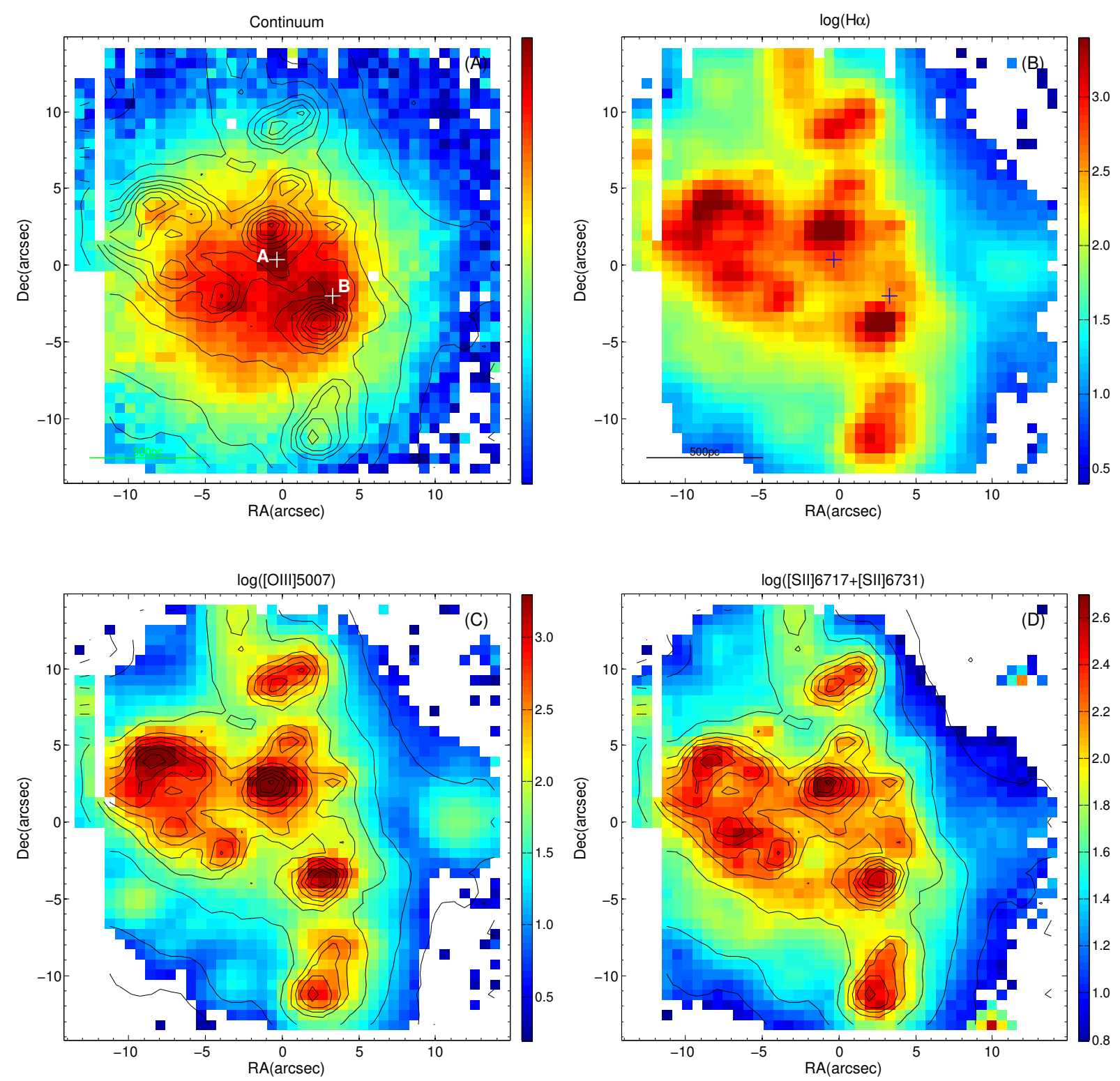

Fig. 2. Panel A) Haro 14 intensity distribution in continuum with $\mathrm{H} \alpha$ contours overplotted; the map has been obtained by summing over the whole orange spectral range, but masking the emission lines. The two major continuum clusters are labeled A and B. The scale in parsec is indicated at the bottom left. The image is scaled in arbitrary flux units. Panel B) $\mathrm{H} \alpha$ emission-line flux (flux units are $10^{-18} \mathrm{erg} \mathrm{s}^{-1} \mathrm{~cm}^{-2}$ ). Panel C) [O III] $\lambda 5007$ emission-line flux with $\mathrm{H} \alpha$ contours overplotted. Panel D) [S II] $\lambda \lambda$ 6717, 6731 emission-line flux with $\mathrm{H} \alpha$ contours overplotted.

content: at least three different populations are identified in this galaxy. We disentangled two distinct episodes of SF in the central region: the very young ionizing stars, i.e., Knots $1-8$ (ages $\leq 10 \mathrm{Myr}$ ), and an intermediate-age population (the HSB clusters visible in the continuum frames, Knots A and B). Both of these stellar components rest on an underlying LSB host galaxy, whose optical and NIR colors indicate an age of several Gyr (Marlowe et al. 1999; Noeske et al. 2003). The properties of the two more recent episodes of SF identified in the galaxy are addressed in the next sections.

\subsection{Extinction and its distribution}

In the optical domain, the interstellar extinction is derived from the ratio of the different $\mathrm{HI}$ Balmer line series to $\mathrm{H} \beta$. The use of IFS data allows us to compute the distribution of the dust across the whole observed FOV.

Figure 4 shows the $\mathrm{H} \alpha / \mathrm{H} \beta$ line-ratio map of Haro 14. The galaxy presents a highly inhomogeneous extinction pattern: the areas of SF are practically dust-free regions $(\mathrm{H} \alpha / \mathrm{H} \beta \lesssim 3)$, whereas the ratio $\mathrm{H} \alpha / \mathrm{H} \beta$ considerable increases when moving toward the periphery of the knots. The largest concentrations of dust are found in the zones of lowest $\mathrm{H} \alpha$ emission, namely, the southeast and western regions. That is consistent with a scenario in which dust is destroyed or swept by the most massive stars.

The extinction coefficient, $\mathrm{C}(\mathrm{H} \beta)$, for every individual spaxel can be derived from the ratio $\mathrm{H} \alpha / \mathrm{H} \beta$ (see Sect. 3.5.2). Typical values of $\mathrm{H} \alpha / \mathrm{H} \beta$ close to the center of the SF knots are about 3 , whereas in the galaxy periphery values of 10 are easily reached. Such ratios imply extinction coefficients $C(\mathrm{H} \beta)=0.06$ and 1.57 , respectively, which translate into magnitudes of extinction in $V$ of $A_{V}=0.12$ and $A_{V}=3.38$, and color excess of $E(B-V)=$ 0.04 and $E(B-V)=1.09^{1}$. Such large variations in $E(B-V)$ stress the importance of obtaining two-dimensional information on the dust distribution even when we are dealing with small and

1 With $A_{V}=2.1 \times C(\mathrm{H} \beta)$ and $E(B-V)=0.697 \times C(\mathrm{H} \beta)$; Dopita \& Sutherland (2003). 


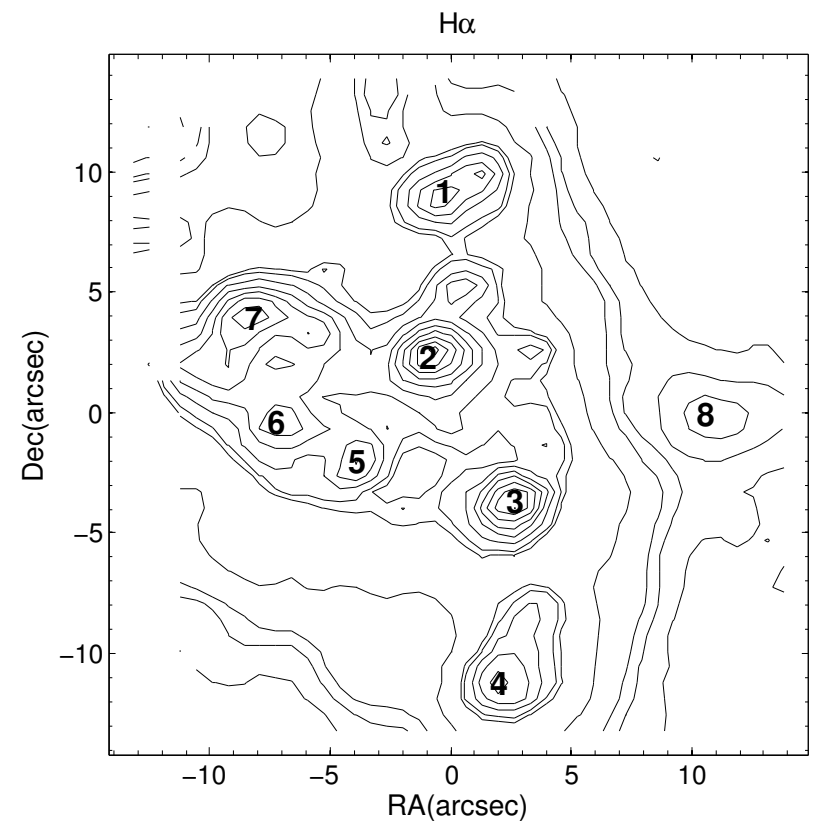

Fig. 3. Contour map of the $\mathrm{H} \alpha$ emission in Haro 14 with the major regions of SF labeled.

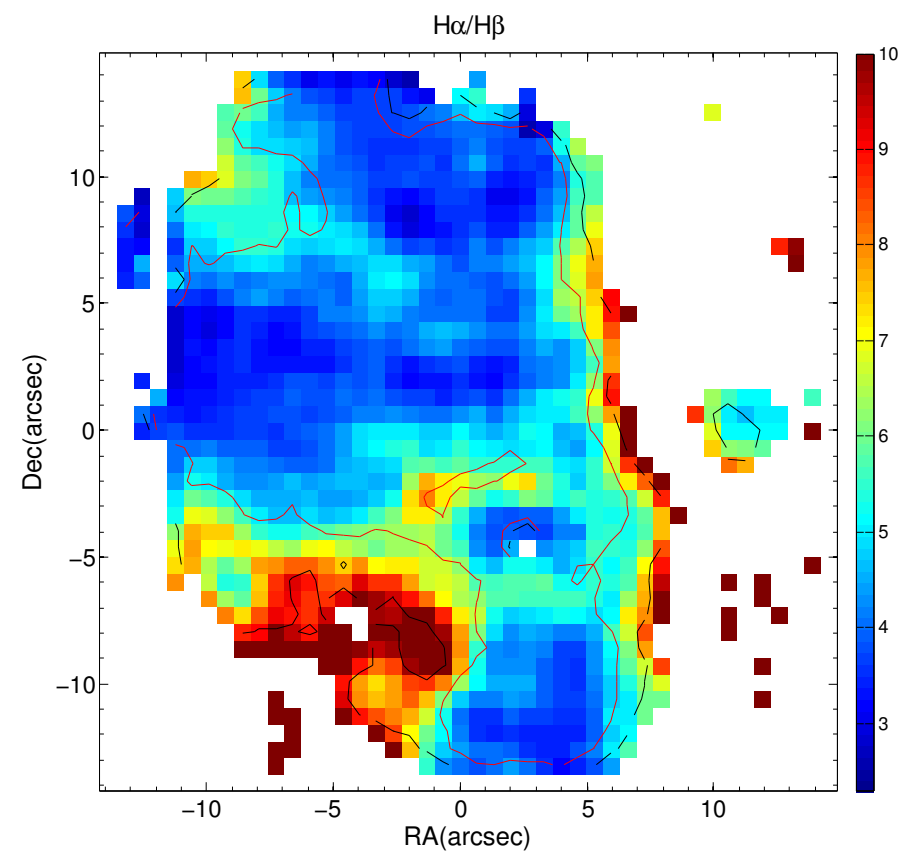

Fig. 4. $\mathrm{H} \alpha / \mathrm{H} \beta$ emission-line ratio for Haro 14 . The map contains only spaxels with values higher than $3 \sigma$ level. Black and red lines delineates the isocontours at $5 \sigma$ level and $10 \sigma$ level, respectively.

low-metal content systems such as dwarfs. The use of an unique extinction coefficient for the whole galaxy (as usual when the spectroscopic data are taken using a slit) can yield large errors in the extinction correction and, hence, in the derived fluxes and magnitudes.

Besides dust, other factors can affect the $\mathrm{H} \alpha / \mathrm{H} \beta$ ratio. In particular, a large component of collisional excitation to the hydrogen lines can enhance $\mathrm{H} \alpha / \mathrm{H} \beta$. That collisional excitation could play a major role in increasing the $\mathrm{H} \alpha / \mathrm{H} \beta$ in the southeastern and western galaxy regions is supported by the fact that the areas of high $\mathrm{H} \alpha / \mathrm{H} \beta$ coincide very well with the regions where

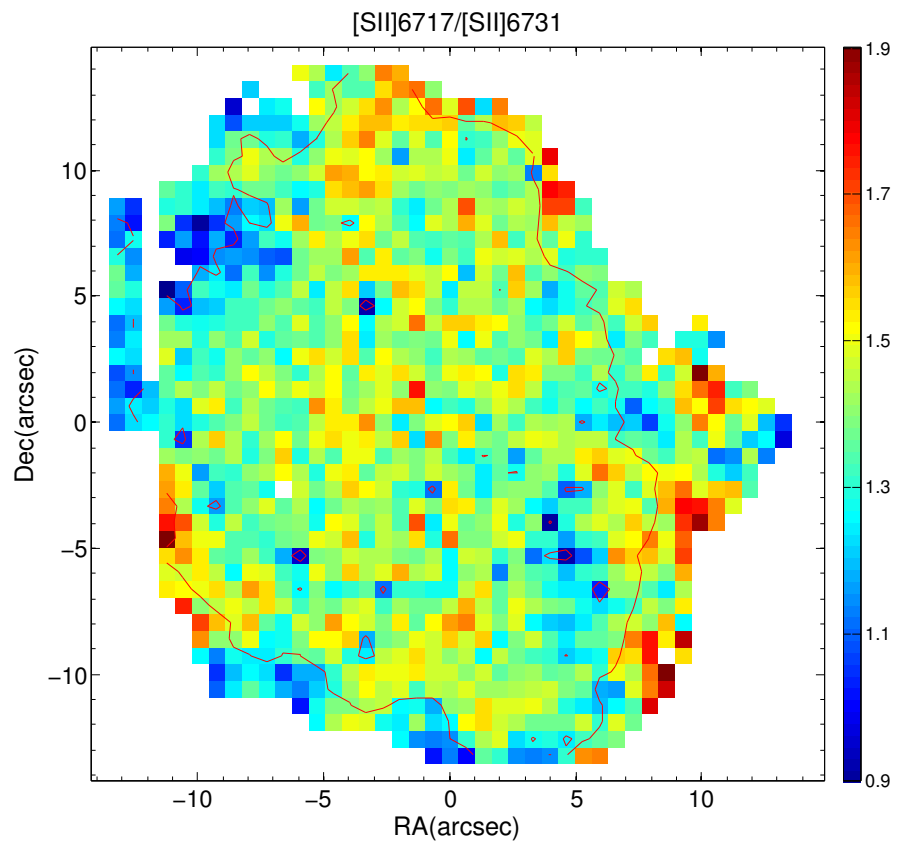

Fig. 5. Electron density sensitive [S II] $\lambda 6717 /[$ S II] $\lambda 6731$ emission-line ratio. The map contains only spaxels with values larger than $3 \sigma$-level. The red line delineates the isocontour at $5 \sigma$-level.

shocks are the dominant ionization mechanism (see Sect. 3.4 and Fig. 11).

\subsection{The electron density distribution}

The electron density, $N_{\mathrm{e}}$, can be derived by observing the effects of collisional deexcitation; the most often used line ratio is [S II] $\lambda 6717 /[\mathrm{S}$ II] $\lambda 6731$, a sensitive density diagnostic in the range 100 to $10000 \mathrm{~cm}^{-3}$.

The central area of the map of [S II] $\lambda 6717 /[\mathrm{S} \mathrm{II}] \lambda 6731$ (Fig. 5) shows only small fluctuations of the line ratio, between 1.5 and 1.2 , which at $T=10000 \mathrm{~K}$ correspond to density in the range $\leq 100 \mathrm{~cm}^{-3}$ up to $240 \mathrm{~cm}^{-3}$. The electron density increases slightly at the galaxy northeast and southwest regions, where values of the line ratio of $0.90 \pm 0.16$ translates into densities of about $900 \mathrm{~cm}^{-3}$.

As expected, the SF knots show mostly low density values $\left(N_{\mathrm{e}} \leq 100 \mathrm{~cm}^{-3}\right)$, whereas the density peaks are reached in the periphery of the knots $(\geq 180-200 \mathrm{pc})$. Areas of higher density at the outskirts of the SF regions are most probably associated with filaments, which are formed when the expanding shell front hits the ISM.

\subsection{On the ionizing mechanism acting in Haro 14}

Two main mechanisms are responsible for the gas ionization in a nebula: photoionization, which is the ionization due to highenergetics photons produced in hot stars or in active galaxy nuclei (AGN), and shock-ionization, which is the collisional ionization that takes place in shocks caused by stellar winds and supernovae (Dopita \& Sutherland 2003).

Extinction-independent line intensity ratios are commonly used to investigate the ionization mechanism in emissionline galaxies (Baldwin et al. 1981; Veilleux \& Osterbrock 1987). The diagnostic line ratios most frequently employed are [O III] $\lambda 5007 / \mathrm{H} \beta$, [N II] $\lambda 6584 / \mathrm{H} \alpha$, [S II] $\lambda \lambda 6717,6731 / \mathrm{H} \alpha$ and [O I] $\lambda 6300 / \mathrm{H} \alpha$. 


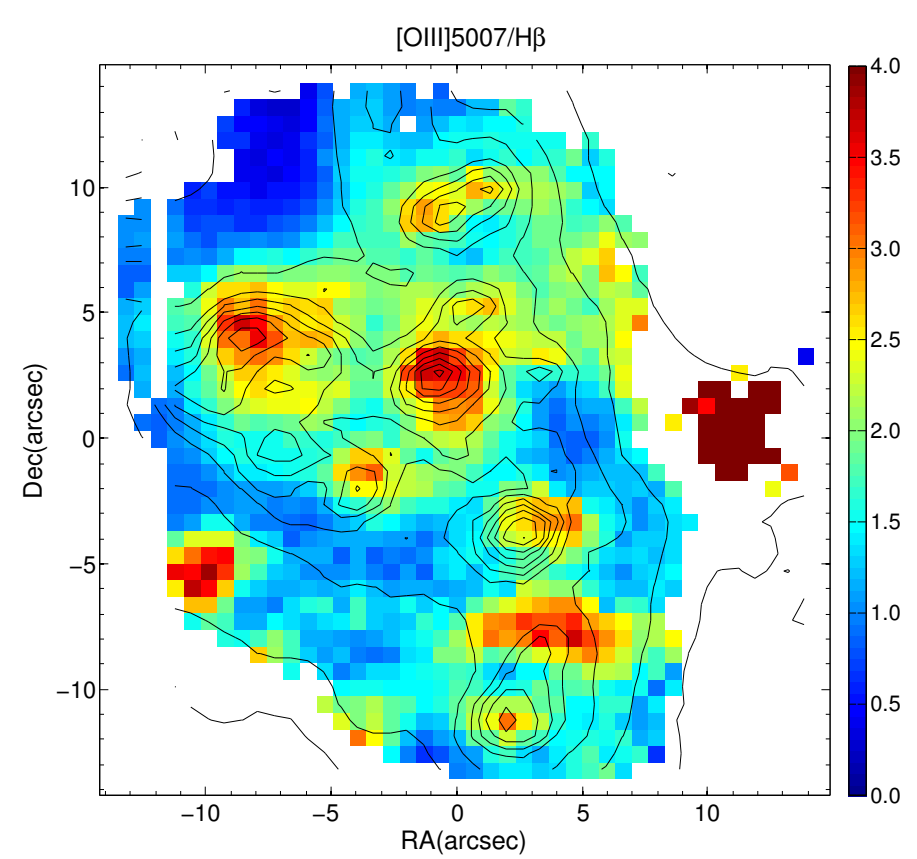

Fig. 6. [O III] $\lambda 5007 / \mathrm{H} \beta$ emission-line ratio map with contours on $\mathrm{H} \alpha$ overplotted.

The two-dimensional information provided by the IFS data allows us to investigate the ionization mechanism acting in Haro 14 across the whole area covered with VIMOS.

The [O III] $\lambda 5007 / \mathrm{H} \beta$ map of Haro 14 displays an intriguing pattern (see Fig. 6). Since [O III] $\lambda 5007$ arises from an ion of relatively high ionization potential $(35.1 \mathrm{eV}),[\mathrm{O} \mathrm{III}] \lambda 5007 / \mathrm{H} \beta$ is a good indicator of the radiation field strength and, as expected, we find that the major SF knots are associated with peaks in [O III] $\lambda 5007 / \mathrm{H} \beta$. However, there are also several other regions in the galaxy, which are not associated with any of the identified H II regions, with very high-excitation values; indeed, the largest excitation (values up to 6) is reached in the Knot 8, the faint blob situated at the west of the galaxy main body. High values of the excitation ([O III] $\lambda 5007 / \mathrm{H} \beta \approx 4$ ) are also found in a clump detected at the galaxy southeast area and, even more puzzling, a patch of high excitation (up to 3.7) is found in the region between Knots 3 and 4 (see Fig. 6).

Several factors can affect the [O III] $\lambda 5007 / \mathrm{H} \beta$ ratio. High values of [O III] $\lambda 5007$ imply a high ionization parameter. Hence in regions photoionized by OB stars, higher excitations are indicative of very high temperatures, and also very hot (very young) OB stars. Alternatively, another ionization mechanism, as AGN or shocks, can be responsible for the high excitation.

The three high-excitation zones in Haro 14, which do not coincide spatially with bright star-forming regions, are (obviously) not powered by an AGN. Although a population of very hot and massive (young) stars can contribute to increase the excitation, Figs. 10 and 11 point to shocks as the most plausible origin of the hard radiation able to produce such high-excitation values.

The [O I] $\lambda 6300 / \mathrm{H} \alpha, \quad[\mathrm{N}$ II] $\lambda 6584 / \mathrm{H} \alpha$, and [S II] $\lambda \lambda 6717,6731 / \mathrm{H} \alpha$ maps are shown in Figs. 7-9. The three maps represent a similar pattern; they trace the regions of SF and present low values in the center of the knots, which increase outward. In the galaxy outskirts, both [O I] $\lambda 6300 / \mathrm{H} \alpha$ and $[\mathrm{S}$ II] $\lambda \lambda 6717,6731 / \mathrm{H} \alpha$ reach values that are inconsistent with photoionization by hot stars ([O I] $\lambda 6300 / \mathrm{H} \alpha \geq 0.1$ and [S II] $\lambda \lambda 6717,6731 / \mathrm{H} \alpha \geq 0.5)$, indicating that another ionizing mechanism, most probably shocks, is acting there.

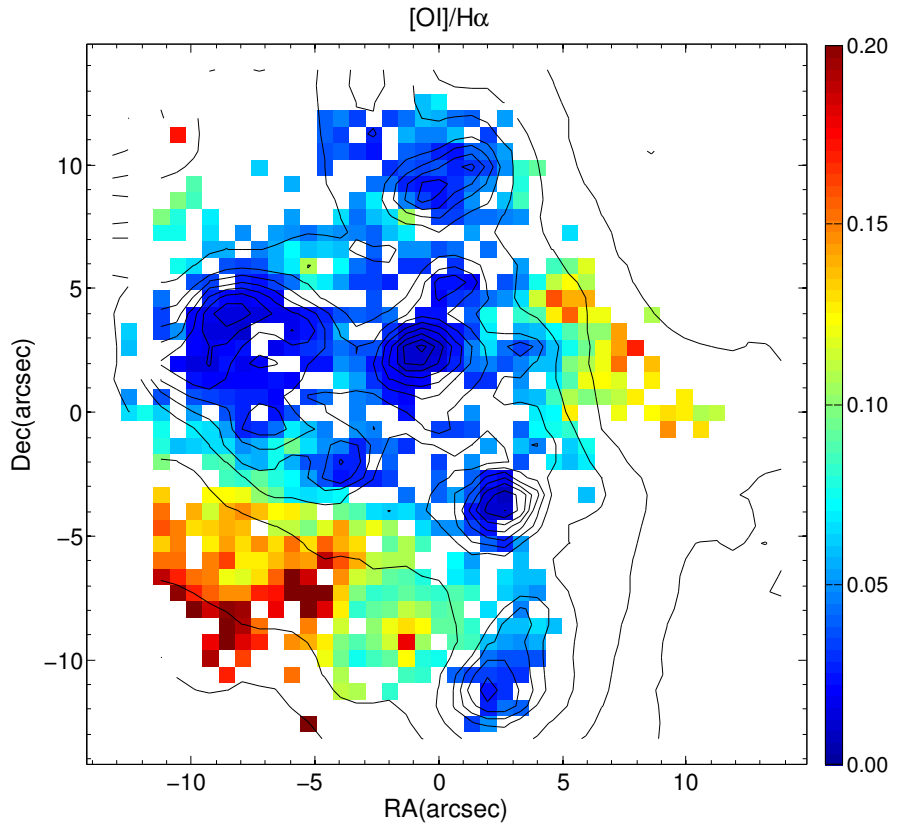

Fig. 7. [OI] $\lambda 6300 / \mathrm{H} \alpha$ emission-line ratio map with contours on $\mathrm{H} \alpha$ overplotted.

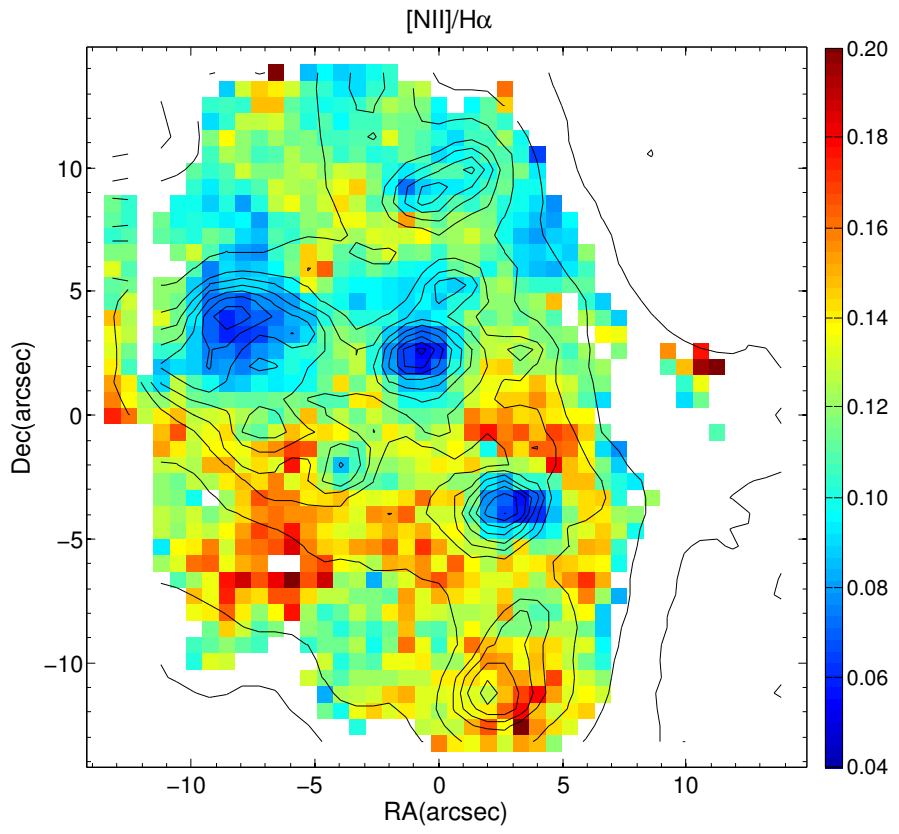

Fig. 8. [N II] $\lambda 6584 / \mathrm{H} \alpha$ emission-line ratio map with contours on $\mathrm{H} \alpha$ overplotted.

To better distinguish among different ionization sources, diagnostic-diagrams are commonly employed (Veilleux \& Osterbrock 1987; Kewley et al. 2001; Kauffmann et al. 2003; Kewley et al. 2006). In combination with two-dimensional spectroscopy, this allows us to probe the power sources acting in different galaxy regions by plotting the spectra from each individual spaxel in the diagnostic diagrams. In recent years this technique has been successfully applied to IFU data (Sharp \& Bland-Hawthorn 2010; Rich et al. 2011, 2012, 2015; Leslie et al. 2014; Belfiore et al. 2015, 2016).

Figure 10 shows the diagnostic-line ratios for the individual spaxels in Haro 14 on the most widely employed diagnostic diagrams, namely, [O III] $\lambda 5007 / \mathrm{H} \beta$ versus [O I] $\lambda 6300 / \mathrm{H} \alpha$, 


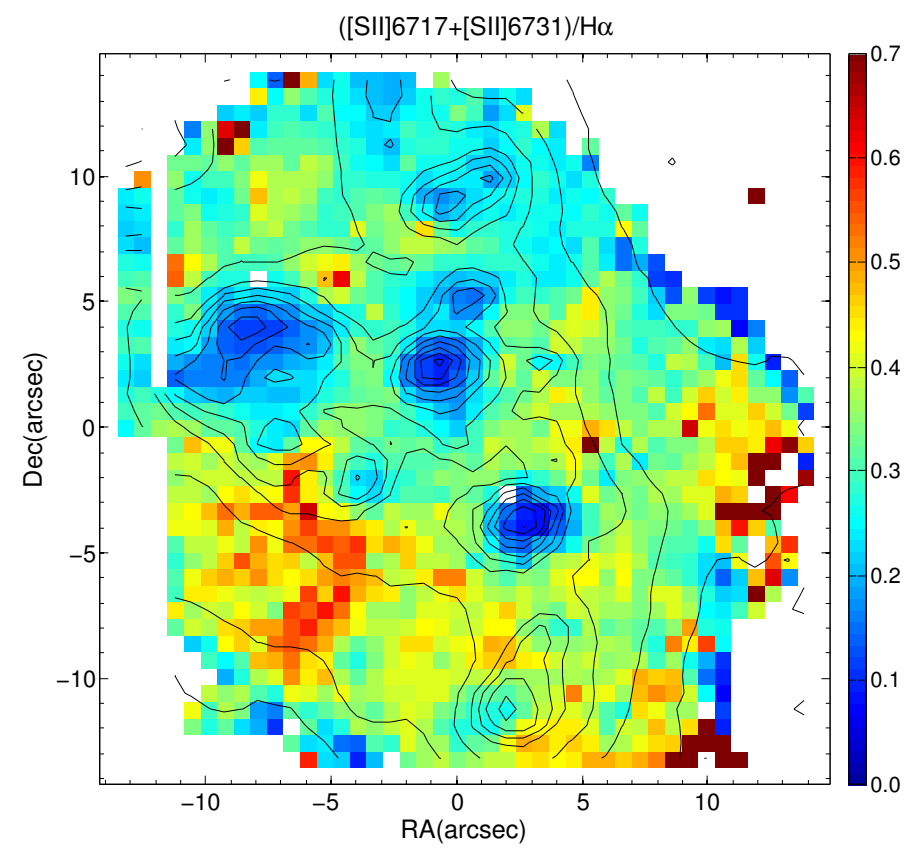

Fig. 9. [S II] $\lambda \lambda 6717,6731 / \mathrm{H} \alpha$ emission-line ratio map with contours on $\mathrm{H} \alpha$ overplotted.

[N II] $\lambda 6584 / \mathrm{H} \alpha$, and [S II] $\lambda \lambda 6717,6731 / \mathrm{H} \alpha$. The maximum starburst line or photoionization line from Kewley et al. (2001) is also shown in the figure. This boundary indicates the limit between gas photoionized by hot stars and gas ionized via other mechanisms; the flux ratios of any object lying above this boundary cannot be modeled by hot stars photoionization, but require an additional contribution from a harder radiation source such as an AGN or shock excitation.

In the [O III] $\lambda 5007 / \mathrm{H} \beta$ versus [S II] $\lambda \lambda 6717,6731 / \mathrm{H} \alpha$, and [O III] $\lambda 5007 / \mathrm{H} \beta$ versus[O I] $\lambda 6300 / \mathrm{H} \alpha$ diagrams a significant number of spaxels fall out of the areas occupied by photoionization for stars. The ratio [N II] $\lambda 6584 / \mathrm{H} \alpha$, which is only weakly dependent on the hardness of the radiation field, but strongly dependent on the metallicity, is not effective at separating shocks from photoionized gas (Allen et al. 2008; Hong et al. 2013).

Figure 11 shows the spatial location in the galaxy of the points plotted in the diagnostic diagrams; the maps are color coded as in Fig. 10, as the redder regions are those that lie further from the maximum starburst line from Kewley et al. (2001). The non-photoionized areas are situated mainly in the galaxy outskirts. In particular, the southwest and west regions lie far above the maximum starburst line, and non-photoionized arc and circular features are visible around the major SF regions. Such an arc of non-photoionized gas, previously seen in starforming galaxies, can be explained as the result of the interaction of two (or more) winds, originated in different stellar clusters. This pattern allows us to conclude with safety that shocks, generated by the mechanical feedback of stellar winds and supernovae explosions, are acting in Haro 14.

\subsection{Integrated spectroscopy}

We identify and delimit the major H II regions and the likely young stellar clusters in Haro 14. Then, we add the spectra of the spaxels in each region to produce a higher $\mathrm{S} / \mathrm{N}$ spectrum from which we determine reliable physical parameters and abundances.
$\mathrm{H}$ II regions are very well traced in $\mathrm{H}$ I recombination-line maps, in which the emission is produced by excited hydrogen atoms decaying to lower levels by radiative transitions. We outline the H II regions in Haro 14 by hand from the $\mathrm{H} \alpha$ maps: this line has a higher $\mathrm{S} / \mathrm{N}$ and is not heavily affected by stellar absorption compared with $\mathrm{H} \gamma$ or $\mathrm{H} \beta$. Young stellar clusters, dominated by hot and blue, but non-ionizing stars, are well visible as HSB regions in continuum maps or bluer regions in color maps. To isolate the young stellar clusters in Haro 14, we made use of the continuum maps. There is not a clear cut criteria to define the limits of these regions. We adopted thence the following approach: we integrated over a boundary that traces the morphology of the clusters, keeping in mind that the minimum size is limited by the seeing; being conservative, we considered only regions with more than 10 spaxels.

We generated integrated spectra of the eight knots identified in the $\mathrm{H} \alpha$ maps (Knots 1 to 8 in Fig. 3), and of the two major HSB stellar clusters seen in continuum (Knots A and B). The main properties of the individual spectra are highlighted below.

All knots show HII regions spectra with strong emission features atop an almost flat continuum, but absorption features around $\mathrm{H} \gamma$ and $\mathrm{H} \beta$ are also seen (see Fig. 12). Residuals from the sky subtraction are evident in several spectra; as explained in Cairós et al. (2015), the subtraction of the sky was a difficult part of the data process. However, these residuals constitute mostly a cosmetic problem because they are located in spectral regions where important features do not fall; mainly the region around [O I] $\lambda 6300$ and the spectral region above $7200 \AA$ in knots 4 and 8 are affected by sky residuals. Fortunately, the line [O I] $\lambda 6300$, redshifted to $6320 \AA$, can also be accurately measured in knots 4 and 8 . Figure 13 shows the [OI] $\lambda 6300$ profile in four of the spectra, including the two more problematic, Knots 4 and 8. It is clear that the line can be reliably measured. Above $7200 \AA$ no lines have been measured in these two knots.

- Knot 1 belongs to the SF chain structure, where it is located about 590 pc to the north of Knot A. It presents a typical nebular spectrum, with prominent Balmer emission lines and strong [O III], [N II], and [S II] forbidden lines atop a weak and rather flat continuum. The flatness of the spectrum and the lack of pronounced absorption features indicates a significant contribution of the nebular continuum.

- Knot 2 is the region where the emission lines are strongest. The conspicuous increase of the continuum toward the blue and the presence of strong absorption wings in the higher terms of the Balmer series, $\mathrm{H} \gamma$ and $\mathrm{H} \beta$, implies a sizable stellar contribution - most probably of hot OB and A stars.

- Knot 3 also belongs to the SF central chain. Its spectrum steps up markedly toward the blue and the presence of an intermediate-age stellar population to the continuum is evidenced by the absorption in the higher order hydrogen Balmer-lines - most probably starlight coming from Knot B, which is displaced only about 130 pc northwest.

- Knot 4, the southeast knot in the central chain structure, is displaced about $725 \mathrm{pc}$ southwest from the continuum peak. Its spectrum reveals strong emission lines on top of an almost flat continuum, in which small absorption wings are in view around $\mathrm{H} \gamma$ and $\mathrm{H} \delta$ in emission.

- Knot 5 is a small HII region, located in the horseshoelike curvilinear structure. The spectrum shows a steep continuum, raising toward the blue and strong absorption on the Balmer lines; absorption lines, such as $\mathrm{CaI} \lambda 4226$ or MgII $\lambda 4481$, are clearly distinguished in the spectrum and 


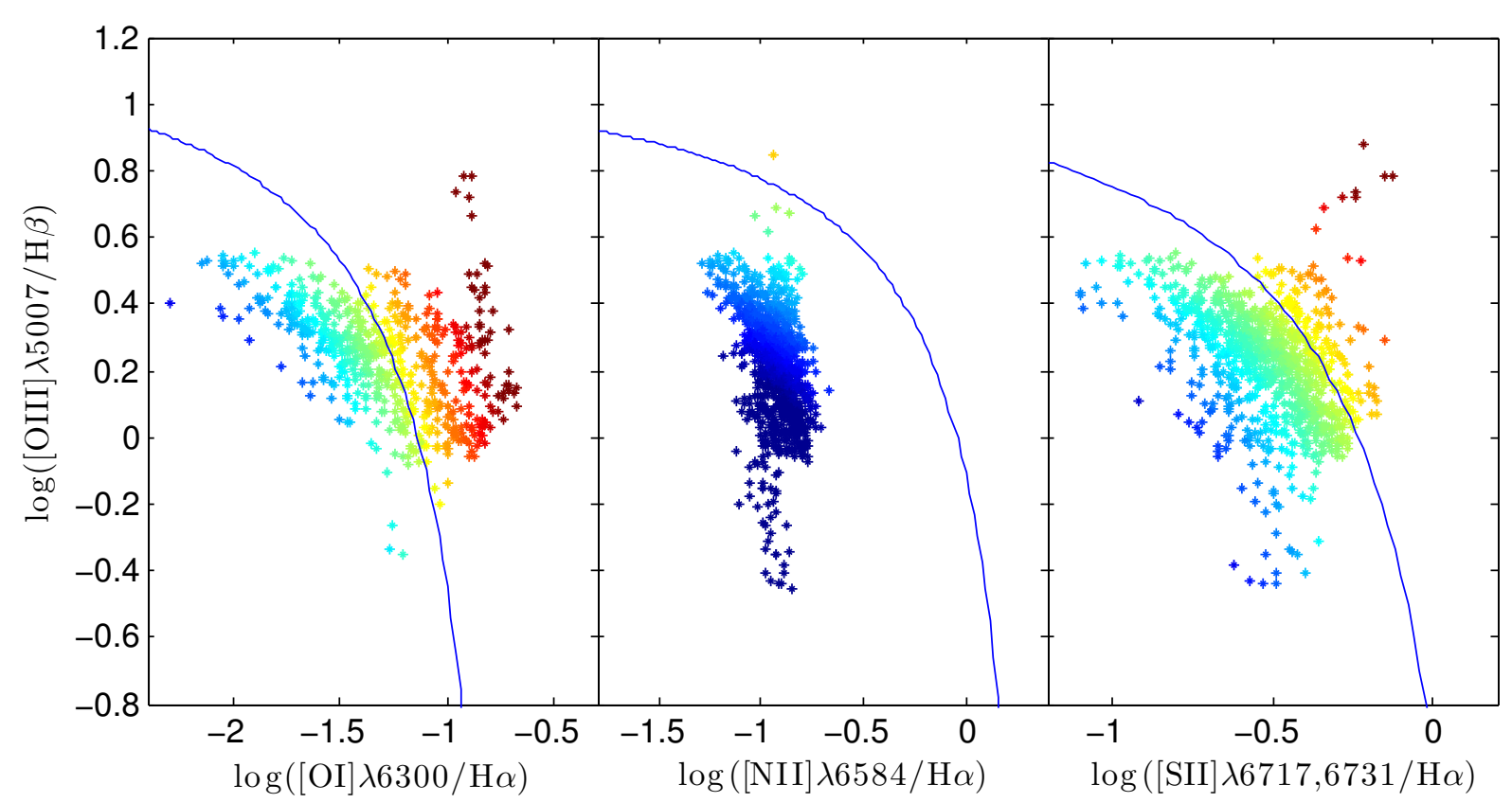

Fig. 10. Optical emission-line diagnostic diagram for the different spaxels in Haro 14. The theoretical maximum starburst line derived by Kewley et al. (2001) is also included in the figure. To better visualize the results on the diagram, the points were color coded according to their distance to the maximum starburst line. In all three diagrams, the lower left section of the plot is occupied by spaxels in which the dominant energy source is radiation from hot stars (blue points in the figure). Additional ionizing mechanisms shift the spaxels to the top right and right part of the diagrams (yellow to red colors).
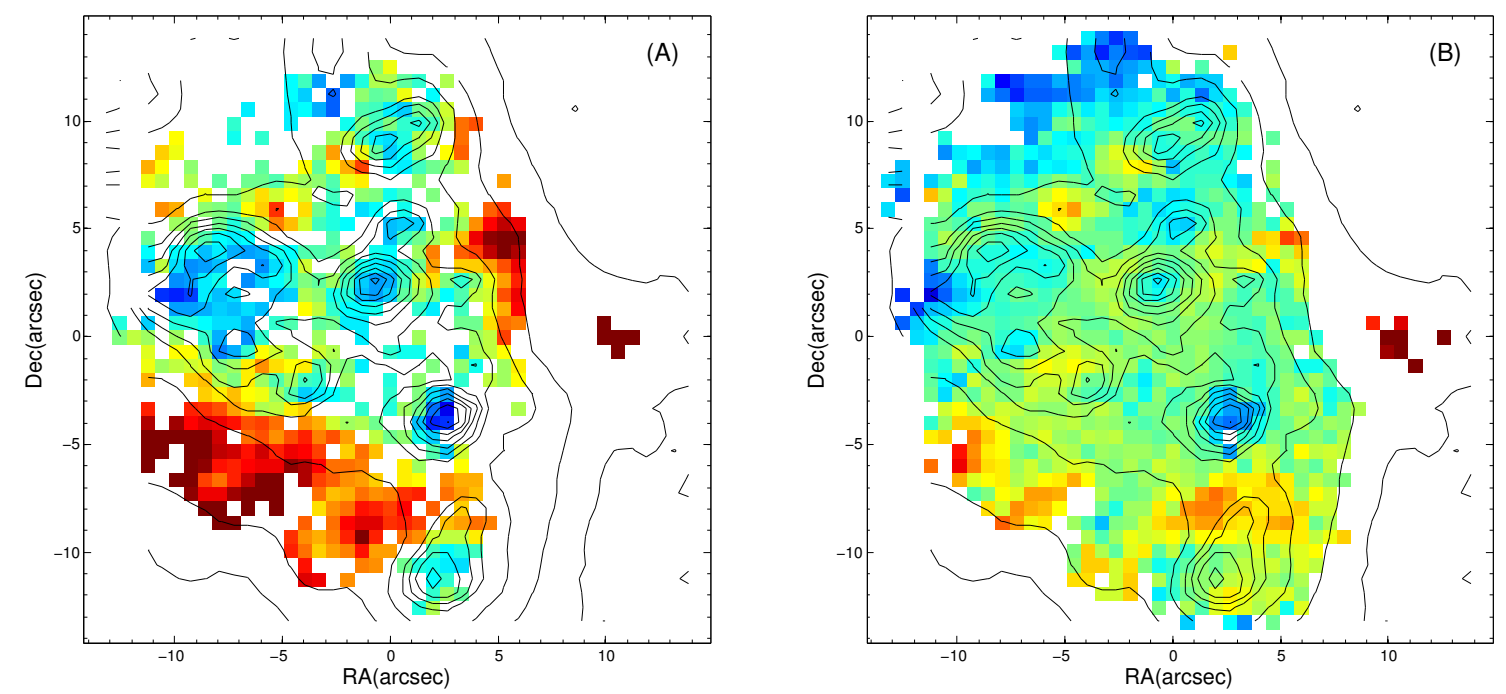

Fig. 11. Spatial localization of the spaxels in the diagnostic diagrams [O III] $\lambda 5007 / \mathrm{H} \beta$ vs. [O I $] \lambda 6300 / \mathrm{H} \alpha$, and [O III] $\lambda 5007 / \mathrm{H} \beta$ vs. [S II] $\lambda \lambda 6717,6731 / \mathrm{H} \alpha$. The color code is the same as in Fig. 10.

are indicative of a significant contribution from intermediateage stars.

- Knot 6 is also a small H II region, located in the loop. As in the case of knot 5 , the spectrum increases notably toward the blue, shows strong absorption wings on the Balmer lines and absorption features ( $\mathrm{CaI} \lambda 4226$ or FeI 14383 ) characteristic of A stars.

- Knot 7 is the largest H II region in the horseshoe-like curvilinear structure; it represents an almost flat spectrum, which increases slightly toward the blue and is dominated by the emission lines of the ionized gas.

- Knot 8 is located on the west, off the galaxy main body. It is a quite faint blob, most clearly seen in the high-excitation [O III] line. It presents a flat spectrum; the poor $\mathrm{S} / \mathrm{N}$ does not allow us to resolve any other feature except the brightest emission-lines.

- Knot $A$ is the peak in continuum. The emission increases visibly toward the blue, and strong absorption wings in the higher order Balmer recombination lines and several absorption features, such as $\mathrm{CaI} \lambda$ 4226, FeI $\lambda$ 4383, or MgII $\lambda 4481$, are detected. The nebular continuum does not significantly contribute to the optical emission, which is instead dominated by stars, indicating that most massive stars have already exploded as supernovae.

- Knot $B$ is a large cluster in which probably several knots are summed together. Although its spatial area comprises the Knot 3 in emission lines, both peaks are displaced about $130 \mathrm{pc}$. The spectrum increases toward bluer wavelengths 
L. M. Cairós and J. N. González-Pérez : Integral field observations of the blue compact galaxy Haro14
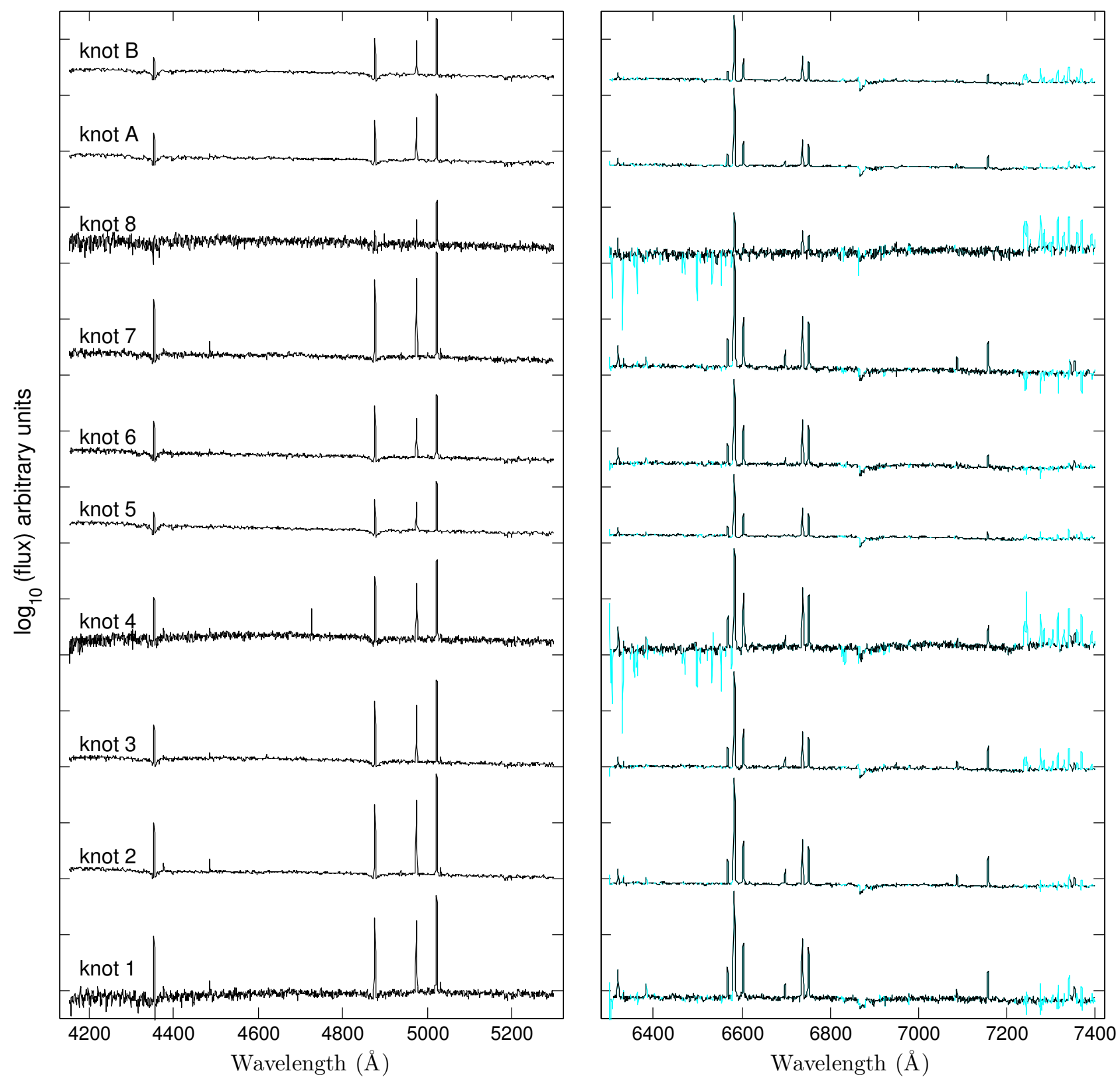

Fig. 12. Flux calibrated spectra of the eight selected H II regions and the two HSB stellar clusters in Haro 14, in logarithmic units. The regions of the spectra affected by the sky lines are plotted in cyan.

and shows marked absorption wings around the Balmer lines in emission.

Figure 12, covering a wide spectral range, does not allow us to resolve individual features. As an example of the absorption features, Fig. 14 presents an enlarged version of particular regions of the knot A, where absorption lines are evident.

\subsubsection{Emission line fluxes}

We have measured the emission-line fluxes in each of the individual spectra using the IRAF $^{2}$ task SPLOT. A Gaussian plus a

\footnotetext{
2 IRAF (Image Reduction and Analysis Facility) is a software system for the reduction and analysis of astronomical data. It is distributed by the NOAO, which is operated by the Association of Universities for Research in Astronomy, Inc., under cooperative agreement with the National Science Foundation
}

linear function were fitted to the line and continuum between two cursor-marked positions. In $\mathrm{H} \gamma$ and $\mathrm{H} \beta$ an underlying absorption is often evident as wide absorption wings, in those cases we fitted two Gaussians, one in absorption and one in emission, where the absorption wings were conspicuous. The equivalent width of $\mathrm{H} \gamma$ and $\mathrm{H} \beta$ in absorption range between 2.5 and $5.0 \AA$ (see Table 2), in good agreement with the predictions of evolutionary synthesis models (Olofsson 1995; González Delgado et al. 1999).

Absorption wings are not visible around $\mathrm{H} \alpha$; we then fixed the equivalent width of $\mathrm{H} \alpha$ in absorption equal to the value found for $\mathrm{H} \beta$, which is a reasonable assumption following the predictions of the models (Olofsson 1995).

\subsubsection{Reddening correction}

We derived the interstellar reddening coefficient making use of the Balmer decrement, i.e., fitting the observed Balmer 

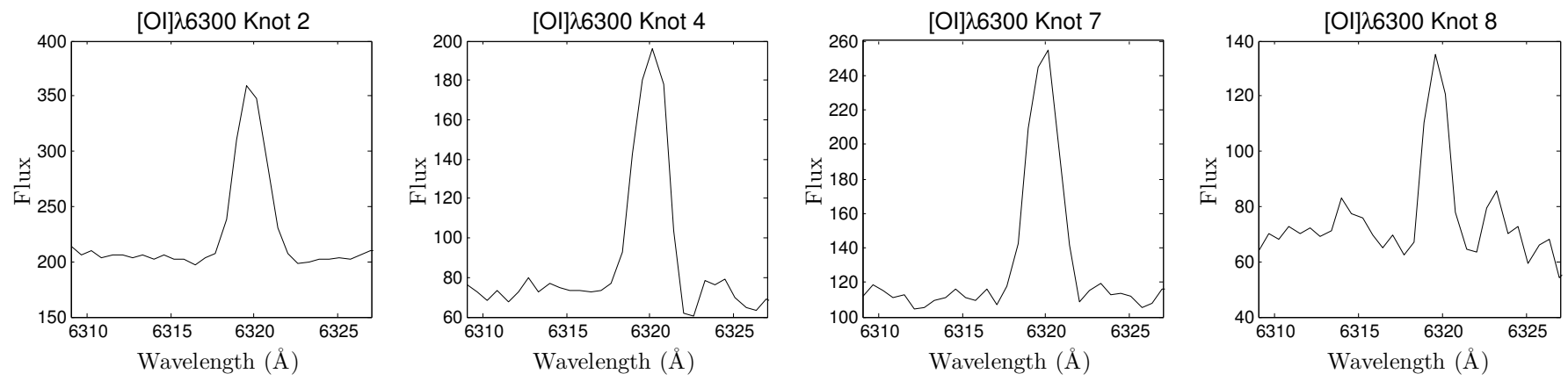

Fig. 13. [O I] $\lambda 6300$ line profile in four of the selected H II regions in Haro 14, including the two regions most heavily affected by sky residuals, knots 4 and 8 . None of the residuals fall above the line, and we can reliably measure its flux.
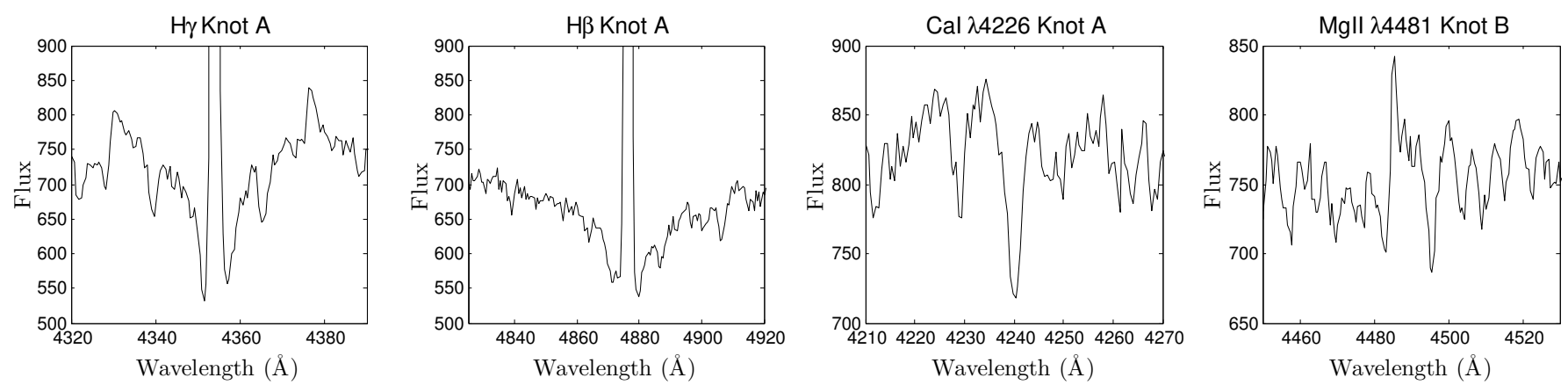

Fig. 14. Some of the absorption features detected in the knot A: $\mathrm{H} \gamma$ and $\mathrm{H} \beta$ absorption wings around $\mathrm{H} \gamma$ and $\mathrm{H} \beta$ in emission, CaI $\lambda 4226$ and MgII $\lambda 4481$.

decrement to its theoretical value. Since the Balmer line intensity ratios are precisely known from atomic theory, any deviation from them must be due to the selective absorption by interstellar dust, which reduces the flux at shorter wavelengths more efficiently than at longer wavelength. The extinction coefficient, $C(\mathrm{H} \beta)$, is determined through the expression

$\frac{F_{\lambda}}{F\left(\mathrm{H}_{\beta}\right)}=\frac{F_{\lambda, 0}}{F\left(H_{\beta, 0}\right)} \times 10^{-C\left(\mathrm{H}_{\beta}\right)} \times\left(f(\lambda)-f\left(\mathrm{H}_{\beta}\right)\right)$,

where $F$ is the flux of any Balmer line in emission, $F_{\lambda, 0} / F\left(\mathrm{H}_{\beta, 0}\right)$ is the ratio of fluxes that would be received on the Earth in absence of interstellar extinction, $F_{\lambda} / F\left(\mathrm{H}_{\beta}\right)$ is the ratio of the fluxes actually observed, and $f(\lambda)$ is the extinction law.

We adopted the theoretical $F(\mathrm{H} \alpha) / F(\mathrm{H} \beta)=2.87$ value, assuming the case $\mathrm{B}$ Balmer recombination decrement in low-density limit, for a temperature of $10000 \mathrm{~K}$ (Osterbrock \& Ferland 2006), and we used the extinction law from O'Donnell (1994). This method assumes that the dust is in a homogeneous obscuring screen, and that all the $\mathrm{H} \alpha$ and $\mathrm{H} \beta$ emission arises from hydrogen recombination and not from collisional excitation.

Although $\mathrm{H} \gamma$ falls into the observed spectral range, we only used the ratio $\mathrm{H} \alpha / \mathrm{H} \beta$ to derive the extinction coefficient because the $\mathrm{H} \gamma$ flux is more severely affected by underlying stellar absorption as compared to $\mathrm{H} \alpha$ or $\mathrm{H} \beta$; the strength of the Balmer series lines in emission decreases rapidly with decreasing wavelength, whereas the equivalent width of the stellar absorption is roughly constant (Olofsson 1995; González Delgado \& Leitherer 1999).

The derived interstellar extinction coefficient, $C(\mathrm{H} \beta)$, and the reddening-corrected intensity ratios are presented in Table 2. For an easier comparison with other data, the number of magnitudes of extinction in $V, A_{V}$, and the color excess, $E(B-V)$, are also shown. It is important to note that different regions show significantly different $C(\mathrm{H} \beta)$, from 0.034 in the Knot B to 0.735 in the Knot A; this translates into increments of magnitudes, $A_{V}$, from 0.074 to 1.589 .

\subsubsection{Diagnostic line ratios, physical parameters, and oxygen abundances}

Values of the diagnostic ratios for the regions selected in Haro 14 are shown in Table 3. Figure 15 shows these values in the diagnostic diagrams [O III] $\lambda 5007 / \mathrm{H} \beta$ versus [N II] $\lambda 6584 / \mathrm{H} \alpha$, [S II] $\lambda \lambda 6717,6731 / \mathrm{H} \alpha$, and [O I] $\lambda 6300 / \mathrm{H} \alpha$. As expected, all the major SF regions fall in the zone of the diagram corresponding to photoionization by hot stars. But Knot 8, the faint blob detected at the galaxy west, lies well above the line delimiting the starburst area on the [O III $] \lambda 5007 / \mathrm{H} \beta$ versus [O I] $\lambda 6300 / \mathrm{H} \alpha$ and [O III] $\lambda 5007 / \mathrm{H} \beta$ versus [S II] $\lambda \lambda 6717,6731 / \mathrm{H} \alpha$ diagrams.

The electronic density and temperature and oxygen abundances of the ionized gas in the individual regions were derived from the reddening-corrected emission-line fluxes.

Electron densities were computed using the [S II] $\lambda \lambda 6717,6731$ ratio (Osterbrock \& Ferland 2006); the derived values are shown in Table 3. The densities in all regions fall in the low-density limit regime $\left(\leq 100 \mathrm{~cm}^{-3}\right)$, except in Knot 7, where it is slightly higher $\left(\approx 100 \mathrm{~cm}^{-3}\right)$.

The electron temperature was calculated from the $[\mathrm{O}$ III $] \lambda 4363 /(\lambda 4959+\lambda 5007)$ ratio in those cases in which reliable values of [O III $] \lambda 4363$ were measured, i.e., Knots 1, 2, and 7 . We follow the procedure described in Izotov et al. (2006). The derived values are shown in Table 3. In Knot 7 where the electronic density is slightly higher, the temperature found is correspondingly lower.

The oxygen abundance cannot be derived using the most reliable direct- $T_{\mathrm{e}}$ method, not even in the knots where the 

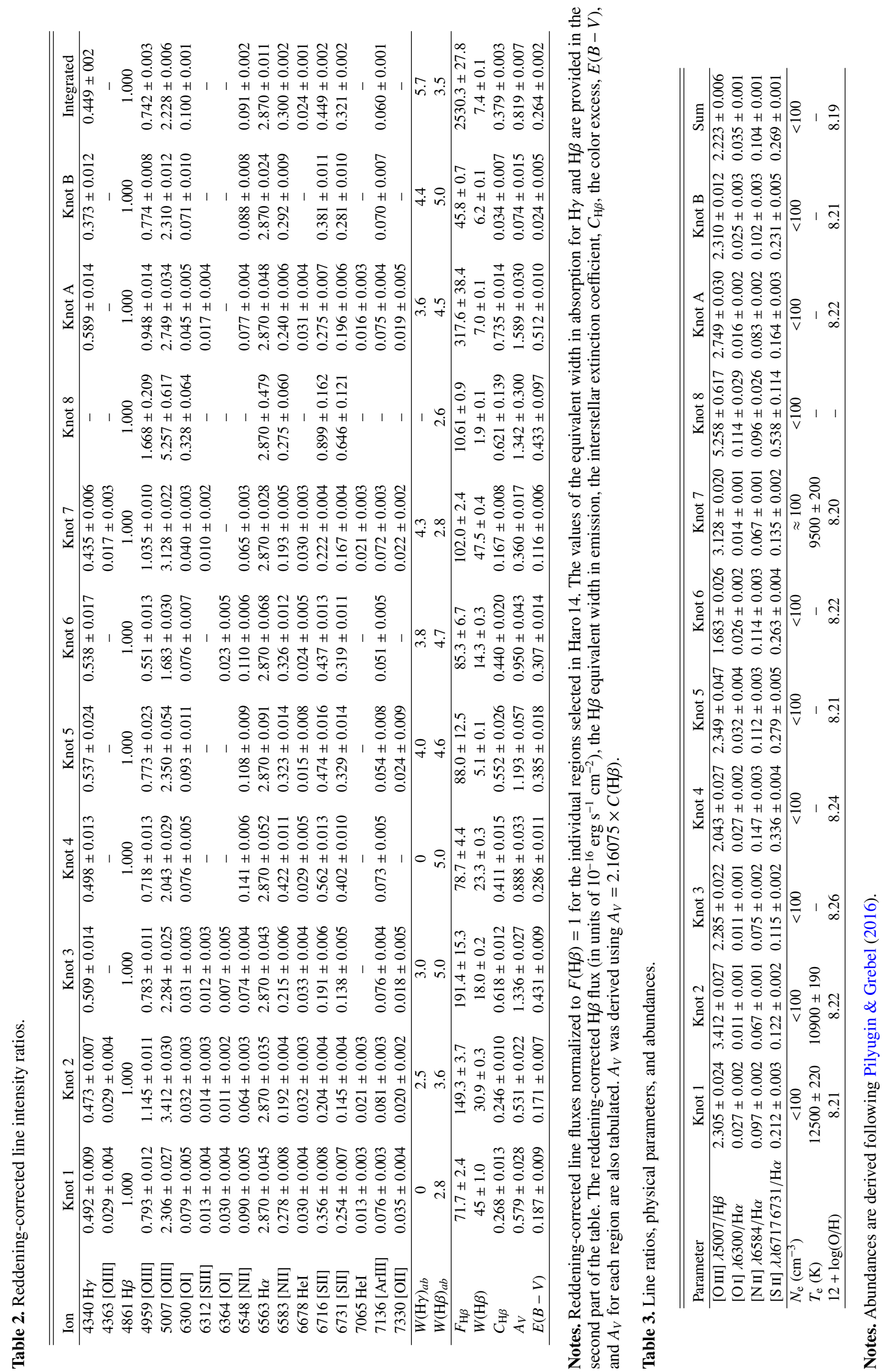


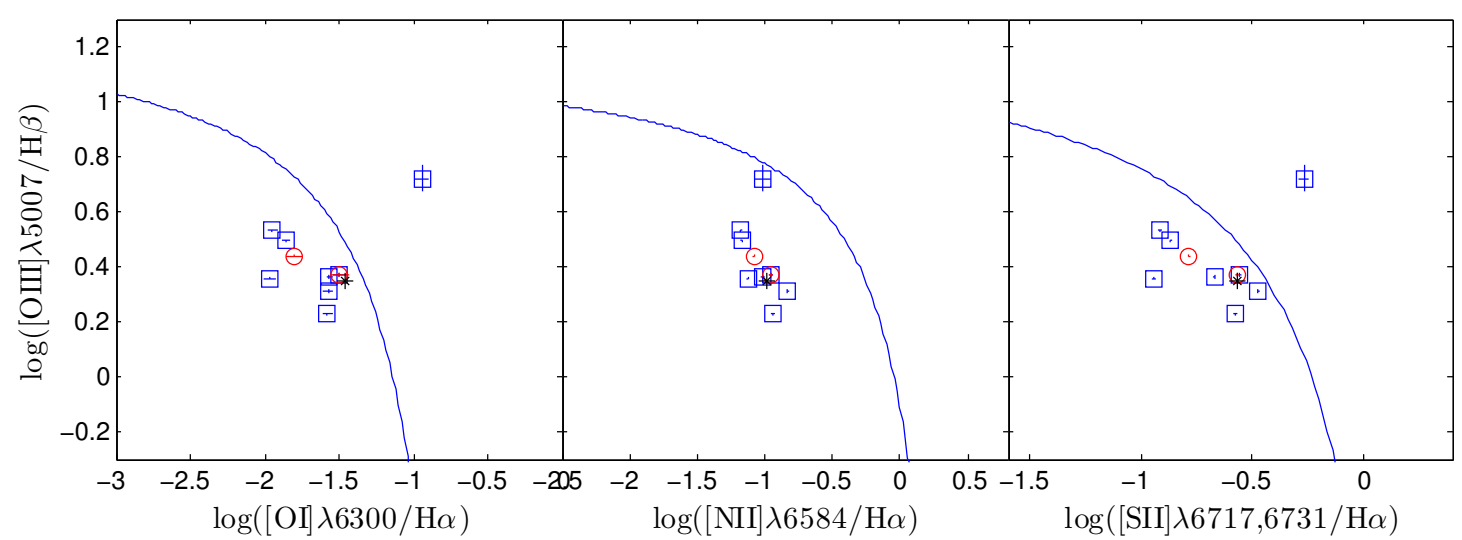

Fig. 15. Optical emission-line diagnostic diagram for the individual regions identified in Haro 14, with the theoretical maximum starburst line (Kewley et al. 2001). Ratio values for the eight SF knots are shown as blue squares, for the two HSB stellar clusters as red circles, and for the integrated spectrum as a black star; errors are plotted as small crosses.

[O III] $\lambda 4363$ has been measured. The direct- $T_{\mathrm{e}}$ method requires the fluxes of the [O II] $\lambda 3727+3729$ lines or, alternatively, the [O II] $\lambda 7320,7331$ lines. The VIMOS observing spectral range does not include the most commonly used [O II] $\lambda 3727+3729$ lines. The [O II] $\lambda 7320,7331$ lines, although within the observed range, fall in the zone of the spectra that is badly affected by sky residuals, which makes it impossible to obtain reliable values of the $[\mathrm{O}$ II] $\lambda 7320$ flux.

Numerous alternative methods were suggested for deriving abundances in those cases for which the direct Te-method is not applicable. We estimated the oxygen abundance using the expressions recently proposed by Pilyugin \& Grebel (2016). These authors provide a calibration that is particularly indicated for cases for which the lines [O II] $\lambda 3727+3729$ lines are not available: the oxygen abundance is derived from the intensities of the strong lines [O III] $\lambda \lambda 4957,5007,[\mathrm{~N}$ II] $\lambda \lambda 6548,6584$, and [S II] $] \lambda 6717,6731$.

Results for the regions selected in Haro 14 are shown in Table 3. No signficant spatial variations in the abundances were found; the relative accuracy of the abundances based on the Pilyugin \& Grebel (2016) calibration is $0.1 \mathrm{dex}$. The derived value for the oxygen abundance, $12+\log (\mathrm{O} / \mathrm{H}) \approx 8.22 \pm 0.1$, is slightly lower than the value of $12+\log (\mathrm{O} / \mathrm{H}) \approx 8.41 \pm 0.02$ reported in Hunter \& Hoffman (1999).

\subsection{Kinematics of the ionized gas}

We studied the kinematics of the ionized gas fitting Gaussian curves to the line profiles of the brightest emission lines, namely, $\mathrm{H} \alpha$ and [O III] 25007 . A significant percentage of the high $\mathrm{S} / \mathrm{N}$ $\mathrm{H} \alpha$ line profiles exhibit a broad low-intensity component and, in those cases, the profiles were optimally fitted using two Gaussians: a bright narrow component (with $\sigma \leq 35 \mathrm{~km} \mathrm{~s}^{-1}$, corrected for instrumental broadening), and the broad low-intensity component ( $80 \mathrm{~km} \mathrm{~s}^{-1} \leq \sigma \leq 150 \mathrm{~km} \mathrm{~s}^{-1}$, also corrected for instrumental effects). The broad component is quite faint and hard to fit even in $\mathrm{H} \alpha$.

The line-of-sight velocity of the emitting gas in $\mathrm{H} \alpha$ (for the bright narrow component) and [O III $] \lambda 5007$, measured from the Doppler shift of the Gaussian line profile centroids relative to the systemic velocity of the galaxy, are shown in Fig. 16. In both maps the velocity distribution presents a complex structure: most regions are moving with respect to the systemic velocity, but the movements do not correspond to a simple rotation. Regions of material are moving toward us in the east and north galaxy areas: at the north, the blueshifted region spatially coincides with the Knot 1 and, at the east, the region traces the periphery of the horseshoe-like curvilinear structure. Such complex velocity fields trace the movements of the gas in the perturbed ISM that is dominated by feedback effects. The amplitude of the velocity field is about $50 \mathrm{~km} \mathrm{~s}^{-1}$. $\mathrm{H} \alpha$ and [O III] $\lambda 5007$ velocity fields display roughly the same pattern, but discrepancies appear on the galaxy west, where some redshifted regions come out in [O III] $\lambda 5007$ but not in $\mathrm{H} \alpha$. Differences between the hydrogen recombination and forbidden line velocities maps can arise because these lines did not originate in the same gas region, and Knot 8 is better distinguished in [O III] $\lambda 5007$.

Figure 16 also shows the line-of-sight velocity dispersion for the bright narrow component of $\mathrm{H} \alpha$ and [O III] $\lambda 5007$. Values of the dispersions are low in the whole covered area, but the map has a clear structure: the regions with higher dispersions $\left(25 \mathrm{~km} \mathrm{~s}^{-1} \leq \sigma \leq 35 \mathrm{~km} \mathrm{~s}^{-1}\right)$ are situated in the periphery of the major SF knots, and are mostly concentrated around a band, which crosses the galaxy in the southeast to northwest direction. Also, shocks dominated areas have higher line-of-sight velocity dispersions and, as expected, H II regions present the lowest dispersion values.

The $\mathrm{H} \alpha$ emission flux map of the broad low-intensity component is shown in Fig. 17. This second component is faint (its luminosity represents about $6 \%$ of the total $\mathrm{H} \alpha$ luminosity), but the structure of its flux distribution confirms that it is indeed a real component and not the result of instrumental effects. The emission is localized in the major regions of SF, being stronger in the knots placed in the periphery of the superbubble feature. The line-of-sight velocity and velocity dispersion for the broad low-intensity component are also shown in Fig. 17.

Multiple components have often been found in BCGs (Östlin et al. 2001; Westmoquette et al. 2008, 2010; James et al. 2009,2013 ) and, in particular, the presence and origin of a lowintensity broad spectral component has been widely discussed. Izotov et al. (2007) conclude, from an analysis of a large number of BCGs exhibiting broad emission lines, that most probably this emission is associated with the evolution of massive stars and their interaction with the circumstellar and ISM. The spatial distribution of the $\mathrm{H} \alpha$ flux (Panel A in Fig. 17) is consistent with this scenario, as the emission is restricted to the major SF regions and their close proximity. The values of the line-of-sight velocity dispersions $\left(80 \mathrm{~km} \mathrm{~s}^{-1} \leq \sigma \leq 150 \mathrm{~km} \mathrm{~s}^{-1}\right.$ ) are also consistent with this broadband feature caused by dynamical processes related to the evolution of massive stars. 
L. M. Cairós and J. N. González-Pérez : Integral field observations of the blue compact galaxy Haro14
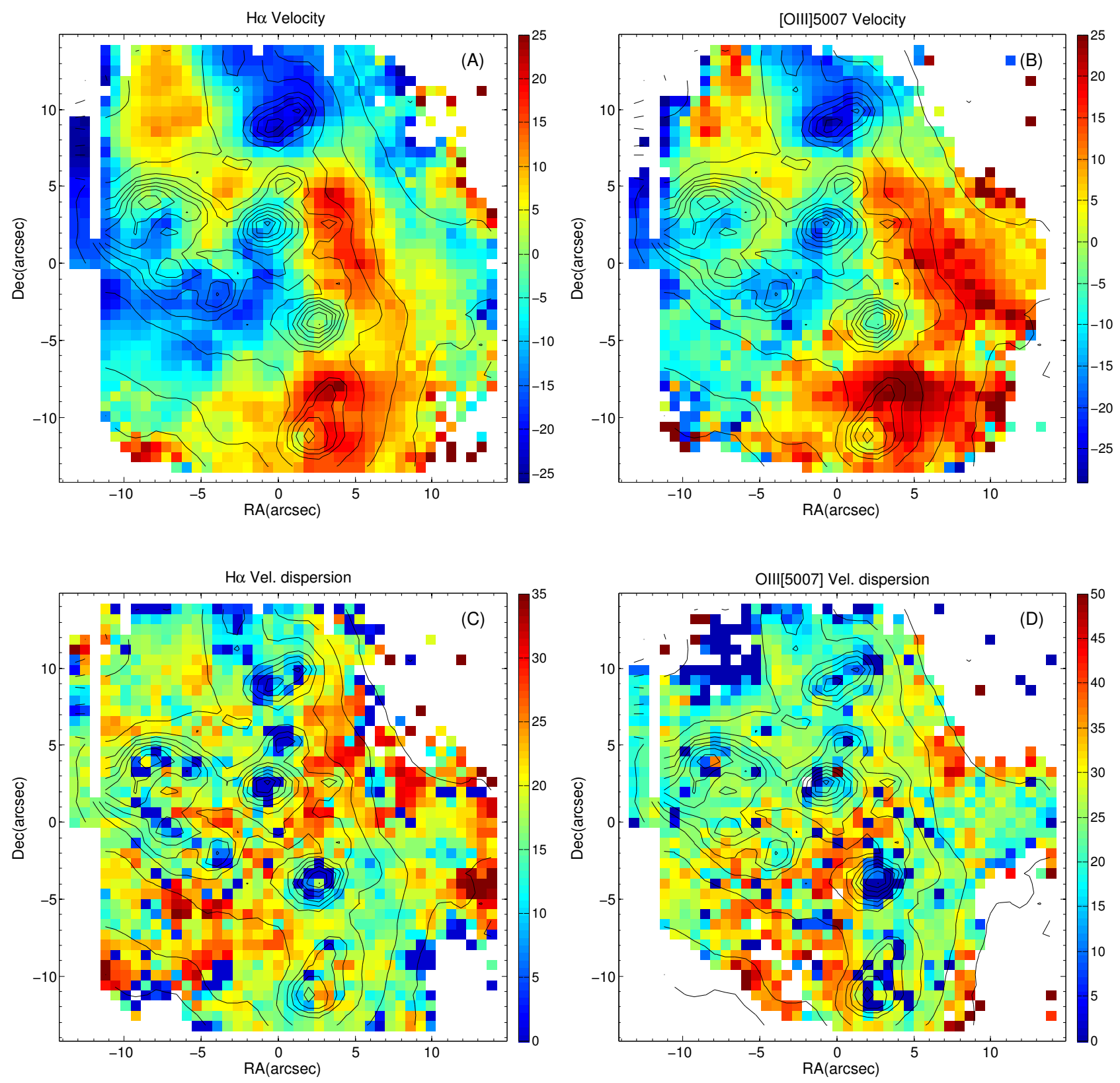

Fig. 16. $\mathrm{H} \alpha$ and [O III] $\lambda 5007$ line-of-sight velocity fields (panels A) and B)) and velocity dispersion maps (panels C) and D)).
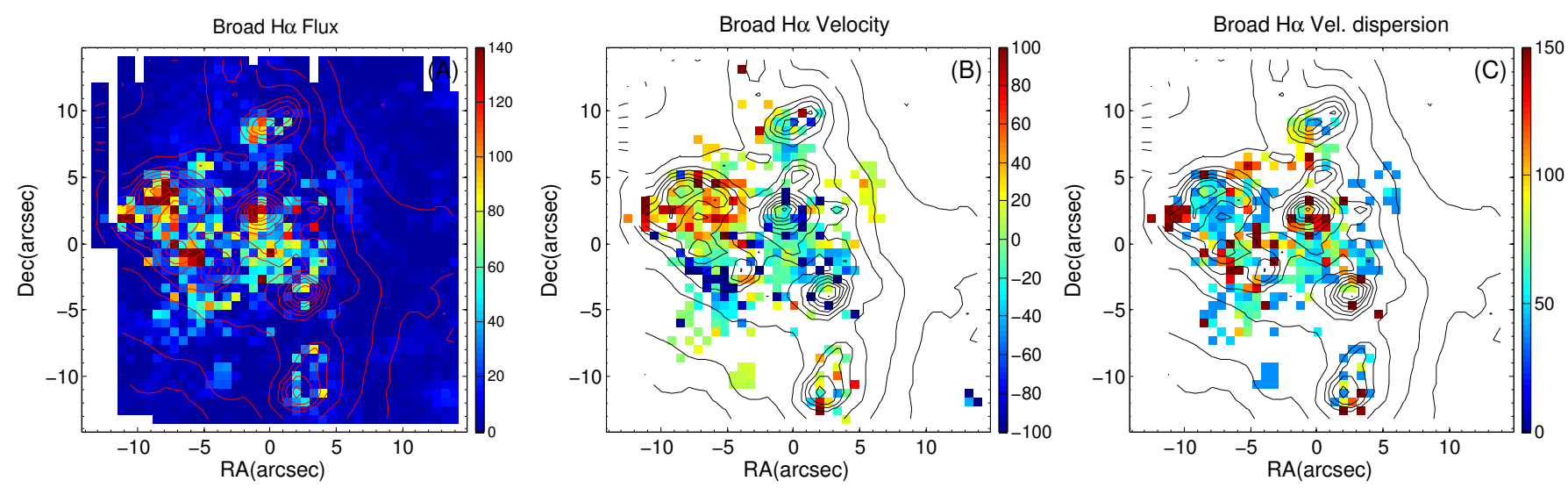

Fig. 17. $\mathrm{H} \alpha$ flux for the low-brightness broad component (panel A)). $\mathrm{H} \alpha$ velocity field for the low-brightness broad component (panel B)). Velocity dispersion for the low-brightness broad component (panel C)). 
Table 4. $\mathrm{H} \alpha$ properties of the SF knots.

\begin{tabular}{cccccccc}
\hline \hline Knot & $\begin{array}{c}\text { Diameter } \\
(\mathrm{pc})\end{array}$ & $\begin{array}{c}F\left(\mathrm{H}_{\alpha}\right) \\
\left(\mathrm{erg} \mathrm{cm}^{-2} \mathrm{~s}^{-1}\right)\end{array}$ & $\begin{array}{c}W\left(\mathrm{H}_{\alpha}\right) \\
(\AA)\end{array}$ & $\begin{array}{c}\log L\left(\mathrm{H}_{\alpha}\right) \\
\left(\mathrm{erg} \mathrm{s}^{-1}\right)\end{array}$ & $\begin{array}{c}S F R \\
\times 10^{-3}\left(M_{\odot} \mathrm{yr}^{-1}\right)\end{array}$ & $\begin{array}{c}N_{\mathrm{LyC}} \\
\left(\mathrm{photons} \mathrm{s}^{-1}\right)\end{array}$ & $\begin{array}{c}\mathrm{Age}_{\mathrm{H}_{\alpha}} \\
\mathrm{Myr}^{50}\end{array}$ \\
\hline 1 & 190 & $205 \pm 4$ & 177 & 38.62 & 3.2 & 3.02 & 5.7 \\
2 & 144 & $428 \pm 6$ & 157 & 38.94 & 6.7 & 6.24 & 5.9 \\
3 & 131 & $550 \pm 17$ & 111 & 39.05 & 8.5 & 3.85 & 6.2 \\
4 & 186 & $226 \pm 6$ & 150 & 38.67 & 3.5 & 3.26 & 5.9 \\
5 & 169 & $253 \pm 13$ & 28 & 38.71 & 3.5 & 3.24 & 8.5 \\
6 & 152 & $245 \pm 9$ & 75 & 38.69 & 3.71 & 3.44 & 6.6 \\
7 & 158 & $292 \pm 3$ & 223 & 38.77 & 4.64 & 4.29 & 5.4 \\
8 & 231 & $30 \pm 9$ & 11 & 37.78 & 0.4 & - & - \\
\hline
\end{tabular}

Notes. $F\left(\mathrm{H}_{\alpha}\right)$ in units of $10^{-16} \mathrm{erg} \mathrm{s}^{-1} \mathrm{~cm}^{-2} . \mathrm{H} \alpha$ fluxes were corrected for interstellar extinction using the values provided in Table 2.

\section{Discussion}

The first step toward establishing the evolutionary status and star-forming history of a galaxy is to disentangle and characterize its different stellar components. The comparison of continuum and emission-line maps provide us with the first insights into the stellar content of Haro 14. Three different stellar populations were distinguished: the very young stars (i.e., Knots 1-7), an intermediate-age stellar population (Knots A and B), and the more extended, redder, and supposedly older underlying stellar component. The small and fainter blobs detected at large galactocentric distances (i.e., Knot 8, see Fig. 3) could be even younger sites of SF.

The stars detected in emission-line maps are young, as only short-lived OB stars, with temperatures $\geq 30000 \mathrm{~K}$ and masses above $10 M_{\odot}$, are able to produce photons with energy large enough to ionize hydrogen $(\geq 13.6 \mathrm{eV})$; because such stars evolve quickly, on timescales of Myr, emission-line maps only trace stars with ages $\leq 10 \mathrm{Myr}$.

Haro 14 emission-line maps revealed SF through the whole mapped area. Using the $\mathrm{H} \alpha$ map we identified and delimited eight major knots: four H II-regions located in the chain-like structure oriented north-south, and another three situated in the rim of the loop, which departs from the central chain and stretches toward east (see Sect. 3.1). At LSB levels several blobs and filaments were barely distinguished; the major blob is located at the galaxy west (Knot 8).

The morphology in continuum and emission lines (Fig. 2) strongly advocate a scenario of triggered SF, with the starforming activity propagating outward. The emission in continuum peaks close to the galaxy center, whereas the maxima in emission lines are reached at larger galactocentric distances. According to this scenario the recent episode of SF would have been triggered by the mechanical energy input from massive stars and supernovae generated in a previous burst (likely the HSB continuum clusters); the knots situated in the loop (namely Knots 5-7) strongly suggest new SF that is taking place in the walls of a large-scale expanding structure (supershell or superbubble), and the fainter knots at larger galactocentric distances could be even younger sites of SF.

Triggered SF occurs when supersonic flows generated by distant supernovae blast waves or stellar winds sweep up a shell of shocked neutral gas; eventually this shell fragments and condenses to produce a new generation of stars (Elmegreen \& Lada 1977). If triggered SF has happened, the separation in time and space of the various episodes of SF must be related in a consistent way. Therefore the first step to verify the scenario of triggered SF in Haro 14 is to constraint the ages of its different episodes of SF, namely, the young H II-regions (Knots 1-8) and the HSB stellar clusters.

Table 4 presents the $\mathrm{H} \alpha$ sizes, fluxes, equivalent widths, and luminosities of the SF knots; their sizes go from 131 up to $231 \mathrm{pc}$, placing them in the range of giant $\mathrm{H}$ II regions. As a reference, 30 Doradus has a diameter of about $300 \mathrm{pc}$; these diameters must be understood only as crude indicators of size. We are limited in resolution and some knots, such as Knot 2, include most probably at least two different SF regions. Indeed, it is not easy to distinguish between large simple H II regions and close clusters of smaller regions, which is beyond the scope of this work. All but Knot 8 show $\mathrm{H} \alpha$ luminosities in the range of giant extragalactic H II regions. Star-forming rates (SFR) and number of ionizing photons, computed from the $\mathrm{H} \alpha$ luminosity following Osterbrock \& Ferland (2006), are also shown.

We used the STARBURST 99 evolutionary synthesis models (Leitherer et al. 1999) to constraint the properties of the knots. We compared the measured $\mathrm{H} \alpha$ equivalent widths with the values predicted by models with metallicity $z=0.008$, which is the value closer to the metallicity derived from the emissionline fluxes, a reasonable approximation to the metallicity of a young population. We found that, in all cases, we can reproduce the equivalent widths with an instantaneous burst of SF and the Salpeter initial mass function (IMF) with an upper mass limit of $100 M_{\odot}$. The ages estimated for each knot are presented in Table 4.

These age estimates are upper limits to the real ages, as the measured equivalent widths of Balmer emission lines are always inferior levels to the true values. Two factors can severely affect the measurement of the equivalent width in emission: first, the contribution of the underlying absorption due to AF stars from the burst, which is included in the emission flux and decreases its final value; and, second, the presence of a higher continuum due to older stars, which dilutes the values of the equivalent width. The decrease of equivalent width in turn results in larger ages.

Measured $\mathrm{H} \alpha$ fluxes were corrected for stellar absorption (see Sect. 3.5.1), but no attempt has been carried out to correct for the effect of a higher continuum; such a correction would require a precise modeling of the older and intermediate-age stellar populations in every spaxel.

Most of the regions present ages around $6 \mathrm{Myr}$, which is consistent with the non-detection of Wolf-Rayet signatures in the integrated spectra of the knots. Only Knot 5 has noticeably larger ages, but the spectrum of this knot shows a substantially high continuum and, at the same time, it is moderate in flux (the larger 
the flux in emission, the less important the effects of absorption and dilution). Therefore, the larger ages found here are most probably due to the effect of the underlying population.

Knots A and B (Fig. 2) do not spatially coincide with any peak in emission lines, indicating that the SF activity has already ceased. Therefore no reliable ages can be derived using equivalent width of Balmer lines in emission, as most of the light is coming from the nearby H II regions. Alternatively, equivalent widths in absorption of the higher order Balmer lines have been shown to be reliable age indicators in post-starburst galaxies (Olofsson 1995; González Delgado et al. 1999). By comparing the equivalent width in absorption of $\mathrm{H} \beta$ and $\mathrm{H} \gamma$ with the predictions of the evolutionary synthesis models by González Delgado et al. (1999), we found ages between 6 and $15 \mathrm{Myr}$ (for an instantaneous burst of solar metallicity) and between 15 and $30 \mathrm{Myr}$ (for an instantaneous burst of $0.05 Z_{\odot}$ ) for Knots A and B. The NIR colors of these knots $(J-H=0.6$ and $H-K_{\mathrm{s}}=0.2$; Noeske et al. 2003) appear as earliest when the NIR becomes dominated by red supergiants, indicating ages between 10 and $30 \mathrm{Myr}$, in good agreement with our findings.

We find ages younger than $6 \mathrm{Myr}$ for the extended starburst in Haro 14, whereas the central HSB clusters are fairly older ages of 10-30 Myr. Adopting an age of 20 Myr for the HSB clusters, we find that a blast wave originated in supernovae explosions must travel with a velocity $v=40 \mathrm{~km} \mathrm{~s}^{-1}$ to ignite a SF event at $\approx 590$ pc (the position of Knot 1) 5.7 Myr ago. To ignite the SF at the position of Knot 2 (at $\approx 168 \mathrm{pc}$ ), a velocity of about $12 \mathrm{~km} \mathrm{~s}^{-1}$ is required. Such velocities are in good agreement with the range of velocities found for expanding shells in dwarf irregular and star-forming galaxies (Walter \& Brinks 1999; Silich et al. 2006; Egorov et al. 2014). These calculations are just a very plain approximation. The real case is obviously much more complex, as many supernovae explosions are taking place in different spatial positions and at different times, and the final superbubbles are the collective effect of all of these explosions.

Knot 8 and the other small condensations detected at the southeast periphery of the field, are also suggestive of a scenario of triggered SF. Their high-excitation values are consistent with very young ages; stellar evolution models predict a drop in [O III] $\lambda 5007 / \mathrm{H} \beta$ for ages $\geq 4$ Myr (Copetti et al. 1986; Stasińska \& Leitherer 1996), as the most massive stars leave the main sequence. And also shocks are expected to increase [O III] $\lambda 5007$ (Raymond et al. 1988). On the other hand, a blast wave originated in the central HSB clusters and traveling with a velocity about $40 \mathrm{~km} \mathrm{~s}^{-1}$ takes about $18 \mathrm{Myr}$ to reach Knot 8 .

A substantial percentage of the galaxy spaxels, mostly the regions situated at the southeast and west, are being ionized by a mechanism other than hot stars (see Fig. 10). This is also in agreement with a scenario of triggered SF because in star-forming regions the most viable mechanisms to drive nonphotoionization processes are shocks from supernovae and massive stars winds (Shull \& McKee 1979; Allen 1999).

Summarizing, we conclude that in Haro 14 we most probably see a feedback mechanism at work, i.e., we are able to distinguish different episodes of sequentially triggered (star-induced) SF driven by mechanical energy input from concentrations of massive stars.

\section{Conclusions}

We present a comprehensive spectrophotometric study of the BCG Haro 14 based on IFS data taken with VIMOS at the VLT. About the central $1.7 \times 1.7 \mathrm{kpc}^{2}$ of Haro 14 was observed in the $4150 \ldots 7400 \AA$ wavelength range with a spatial sampling of 0 '.66 per spaxel.

From these data, we build maps in continuum and in the brighter emission lines (e.g., $\mathrm{H} \beta$, [O III] $\lambda 5007, \mathrm{H} \alpha$, [N II] $\lambda 6584$, or $[\mathrm{S} \mathrm{II}] \lambda \lambda 6717,6731)$, which provide information about the galaxy morphology and give insights into its stellar content; produce the $\mathrm{H} \alpha / \mathrm{H} \beta$ Balmer decrement map, which depicts the dust; build the [S II] $\lambda 6717 /[\mathrm{S}$ II] $\lambda 6731$ line-ratio map, which traces the electronic density; construct diagnostic line-ratio maps, namely, [O III] $\lambda 5007 / \mathrm{H} \beta$, [N II] $\lambda 6584 / \mathrm{H} \alpha$, [S II] $\lambda \lambda 6717,6731 / \mathrm{H} \alpha$, and [O I] $\lambda 6300 / \mathrm{H} \alpha$, which are used together with diagnostic diagrams to carry out an extensive investigation of the nebula ionization and excitation; generate the integrated spectrum of the major H II regions and young stellar clusters identified in the maps to determine reliable physical parameters and oxygen abundances; and, finally, build the velocity and velocity dispersion fields, which trace the ionized gas kinematics. The analysis of these datasets allows us to derive the following conclusions:

- Haro 14 presents significant deviations between its stellar and ionized gas distribution. In continuum the intensity peaks close to the galaxy center, where two major stellar clusters have been identified - Knots A and B. In emission line, maxima are distributed in the whole mapped area, where the major SF regions are placed along a linear (chainlike) structure, elongated in the north-south direction, and in a horseshoe-like curvilinear feature, which departs from the central region and extends about 12'!0 (760 pc) eastward; several fainter lumps, better distinguished in the highionization [O III] $\lambda 5007$ map, come out around the major H II regions, at distances about $0.7-0.8 \mathrm{kpc}$ from the galaxy center; the largest (Knot 8) is located at the west.

Using the $\mathrm{H} \alpha$ maps we delimited eight major SF knots, none of which is cospatial with the continuum peaks. This morphology is suggestive of a mechanism of triggered SF taking place in Haro 14.

- We disentangle two different episodes of SF in the central regions of Haro 14. By comparing with evolutionary synthesis models, we constrain their ages: the recent starburst (Knots 1-7) present ages $\leq 6 \mathrm{Myr}$, whereas the HSB stellar clusters (Knots A and B) are appreciably older, with ages between 10 and 30 Myr. These stellar components rest on a several Gyr old underlying LSB host galaxy (Marlowe et al. 1999; Noeske et al. 2003).

- The galaxy presents a highly inhomogeneous $\mathrm{H} \alpha / \mathrm{H} \beta$ lineratio pattern, in which the SF regions are practically dust free, whereas very large $\mathrm{H} \alpha / \mathrm{H} \beta$ values appear in the periphery of the knots; the color excess varies from $E(B-V)=0.04$ up to $E(B-V)=1.09$, when moving from the SF center to the galaxy outskirts. Such large variations in $E(B-V)$ point out the importance of obtaining two-dimensional information on the dust distribution: the use of an unique extinction coefficient for the whole galaxy (as usually done when the spectroscopic data are taken using a slit) can yield large errors in the extinction correction and, hence, in the derived fluxes and magnitudes.

- Diagnostic maps and diagnostic diagrams reveal the presence of a mechanism other than photoionization by hot stars in a significant area of the galaxy. Shocks are the dominant ionizing mechanism in the galaxy southeast and western regions, and also shock-ionized ring and arc features are found surrounding the major SF knots. 
- The excitation map reveal an interesting pattern; the SF knots show, as expected, high-excitation values, but there are also three other zones with very high excitation ([O III $] \lambda 5007 / \mathrm{H} \beta$ up to six), which do not spatially coincide with any of the major knots. Two of these high-excitation regions are associated with faint blobs seen in the galaxy outskirts, and the third is an arc feature located at the south of the chain-like structure, between Knots 3 and 4. These three regions are dominated by shocks. Also very young ages can contribute to such high excitations.

- We produce the integrated spectrum of the eight major SF regions and the two HSB stellar clusters; using these spectra we derive reliable physical properties and oxygen abundances of the 10 selected knots. Electronic densities are found to be low $\left(N_{\mathrm{e}} \leq 100 \mathrm{~cm}^{-3}\right)$ in all the regions but in Knot 7, which presents values slightly higher $\left(N_{\mathrm{e}} \approx 100 \mathrm{~cm}^{-3}\right)$. We derived an oxygen abundance of $12+\log (\mathrm{O} / \mathrm{H}) \approx 8.22 \pm 0.1$ and found no evidence for any significant chemical abundance gradient.

- The kinematics of the warm ionized gas was studied by fitting Gaussian curves to the line profiles of the brightest emission lines, namely, $\mathrm{H} \alpha$ and [O III] $\lambda 5007$. The velocity distribution presents a complex structure with regions of material moving toward us in the east and north galaxy areas. Such complex velocity fields trace the movements of the gas in a perturbed ISM, dominated by feedback effects. Ages derived for the current SF episode (around $6 \mathrm{Myr}$ ) are consistent with the energetics of the ISM dominated by core-collapse TypeII supenovae explosions. The amplitude of the velocity field is about $50 \mathrm{~km} \mathrm{~s}^{-1}$.

In conclusion, the information derived from the VIMOS data allows us to probe the recent star-forming history of Haro 14 . We disentangle two temporally and spatially separated star-forming episodes: the recent starburst (Knots 1-7) and the HSB stellar clusters (Knots A and B). The morphological pattern, the irregular velocity field, dominated by the movement of the ionized gas, and the presence of shocks, all point to a scenario of triggered (star-induced) star formation. The ages of the different episodes of SF, $\approx 6$ Myr for the extended starburst in Haro 14 and about 10-30 Myr for the central HSB clusters, are consistent with the ongoing burst being triggered by the collective action of stellar winds and supernovae that originated in the central HSB stellar clusters.

Acknowledgements. L.M.C. acknowledges support from the Deutsche Forschungsgemeinschaft (CA 1243/1-1). The authors are very grateful to N. Caon and P. Weilbacher, members of the IFU-BCG project. We also thank R. Manso-Sainz for many constructive discussions and comments, and R. Mettin for a careful reading of the manuscript. Further thanks goes to Oliver Mettin for extremely stimulating discussions. This research has made use of the NASA/IPAC Extragalactic Database (NED), which is operated by the Jet Propulsion Laboratory, Caltech, under contract with the National Aeronautics and Space Administration.

\section{References}

Allen, M. L. 1999, Ph.D. Thesis, University of Toronto, Canada Allen, M. G., Groves, B. A., Dopita, M. A., Sutherland, R. S., \& Kewley, L. J. 2008, ApJS, 178, 20

Baldwin, J. A., Phillips, M. M., \& Terlevich, R. 1981, PASP, 93, 5

Belfiore, F., Maiolino, R., Bundy, K., et al. 2015, MNRAS, 449, 867

Belfiore, F., Maiolino, R., Maraston, C., et al. 2016, MNRAS, 461, 3111

Cairós, L. M., Caon, N., Papaderos, P., et al. 2009a, ApJ, 707, 1676
Cairós, L. M., Caon, N., Zurita, C., et al. 2009b, A\&A, 507, 1291 Cairós, L. M., Caon, N., Zurita, C., et al. 2010, A\&A, 520, A90 Cairós, L. M., Caon, N., García Lorenzo, B., et al. 2012, A\&A, 547, A24 Cairós, L. M., Caon, N., \& Weilbacher, P. M. 2015, A\&A, 577, A21 Copetti, M. V. F., Pastoriza, M. G., \& Dottori, H. A. 1986, A\&A, 156, 111 Dekel, A., \& Silk, J. 1986, ApJ, 303, 39

de Vaucouleurs, G., de Vaucouleurs, A., Corwin, Jr., H. G., et al. 1991, Third Reference Catalogue of Bright Galaxies, eds. G. de Vaucouleurs, A. de Vaucouleurs, H. G. Jr. Corwin, R. J. Buta, G. Paturel, \& P. Fouque (Berlin, New York: Springer)

Dopita, M. A., \& Sutherland, R. S. 2003, Astrophysics of the diffuse Universe (Berlin, New York: Springer)

Doublier, V., Caulet, A., \& Comte, G. 1999, A\&AS, 138, 213

Doublier, V., Caulet, A., \& Comte, G. 2001, A\&A, 367, 33

Egorov, O. V., Lozinskaya, T. A., Moiseev, A. V., \& Smirnov-Pinchukov, G. V. 2014, MNRAS, 444, 376

Elmegreen, B. G., \& Lada, C. J. 1977, ApJ, 214, 725

Gil de Paz, A., \& Madore, B. F. 2005, ApJS, 156, 345

Gil de Paz, A., Madore, B. F., \& Pevunova, O. 2003, ApJS, 147, 29

González Delgado, R. M., \& Leitherer, C. 1999, ApJS, 125, 479

González Delgado, R. M., Leitherer, C., \& Heckman, T. M. 1999, ApJS, 125, 489

Grimes, J. P., Heckman, T., Strickland, D., et al. 2007, ApJ, 668, 891

Hong, S., Calzetti, D., Gallagher, III, J. S., et al. 2013, ApJ, 777, 63

Hunter, D. A., \& Elmegreen, B. G. 2006, ApJS, 162, 49

Hunter, D. A., \& Hoffman, L. 1999, AJ, 117, 2789

Izotov, Y. I., Stasińska, G., Meynet, G., Guseva, N. G., \& Thuan, T. X. 2006, A\&A, 448, 955

Izotov, Y. I., Thuan, T. X., \& Guseva, N. G. 2007, ApJ, 671, 1297

James, B. L., Tsamis, Y. G., Barlow, M. J., et al. 2009, MNRAS, 398, 2

James, B. L., Tsamis, Y. G., Walsh, J. R., Barlow, M. J., \& Westmoquette, M. S. 2013, MNRAS, 430, 2097

Kauffmann, G., Heckman, T. M., Tremonti, C., et al. 2003, MNRAS, 346, 1055

Kewley, L. J., Dopita, M. A., Sutherland, R. S., Heisler, C. A., \& Trevena, J. 2001, ApJ, 556, 121

Kewley, L. J., Groves, B., Kauffmann, G., \& Heckman, T. 2006, MNRAS, 372, 961

Kunth, D., \& Östlin, G. 2000, A\&ARv, 10, 1

Le Fèvre, O., Saisse, M., Mancini, D., et al. 2003, in Instrument Design and Performance for Optical/Infrared Ground-based Telescopes, eds. M. Iye, \& A. F. M. Moorwood, Proc. SPIE, 4841, 1670

Leitherer, C., Schaerer, D., Goldader, J. D., et al. 1999, ApJS, 123, 3

Leslie, S. K., Rich, J. A., Kewley, L. J., \& Dopita, M. A. 2014, MNRAS, 444, 1842

Loose, H.-H., \& Thuan, T. X. 1986, in Star-forming Dwarf Galaxies and Related Objects, 73

Marlowe, A. T., Meurer, G. R., Heckman, T. M., \& Schommer, R. 1997, ApJS, 112,285

Marlowe, A. T., Meurer, G. R., \& Heckman, T. M. 1999, ApJ, 522, 183

McCall, M. L., Rybski, P. M., \& Shields, G. A. 1985, ApJS, 57, 1

Moustakas, J., \& Kennicutt, Jr., R. C. 2006, ApJS, 164, 81

Noeske, K. G., Papaderos, P., Cairós, L. M., \& Fricke, K. J. 2003, A\&A, 410, 481

O’Donnell, J. E. 1994, ApJ, 422, 158

Olofsson, K. 1995, A\&AS, 111, 57

Osterbrock, D. E., \& Ferland, G. J. 2006, Astrophysics of gaseous nebulae and active galactic nuclei, 2nd. edn., eds. D.E. Osterbrock, \& G.J. Ferland (Sausalito, CA: University Science Books)

Östlin, G., Amram, P., Bergvall, N., et al. 2001, A\&A, 374, 800

Pilyugin, L. S., \& Grebel, E. K. 2016, MNRAS, 457, 3678

Popescu, C. C., \& Hopp, U. 2000, A\&AS, 142, 247

Raymond, J. C., Hester, J. J., Cox, D., et al. 1988, ApJ, 324, 869

Rich, J. A., Kewley, L. J., \& Dopita, M. A. 2011, ApJ, 734, 87

Rich, J. A., Torrey, P., Kewley, L. J., Dopita, M. A., \& Rupke, D. S. N. 2012, ApJ, 753, 5

Rich, J. A., Kewley, L. J., \& Dopita, M. A. 2015, ApJS, 221, 28

Sharp, R. G., \& Bland-Hawthorn, J. 2010, ApJ, 711, 818

Shull, J. M., \& McKee, C. F. 1979, ApJ, 227, 131

Silich, S., Lozinskaya, T., Moiseev, A., et al. 2006, A\&A, 448, 123

Springel, V., Frenk, C. S., \& White, S. D. M. 2006, Nature, 440, 1137

Stasińska, G., \& Leitherer, C. 1996, ApJS, 107, 661

Tenorio-Tagle, G., \& Bodenheimer, P. 1988, ARA\&A, 26, 145

Thuan, T. X., \& Martin, G. E. 1981, ApJ, 247, 823

Veilleux, S., \& Osterbrock, D. E. 1987, ApJS, 63, 295

Walter, F., \& Brinks, E. 1999, AJ, 118, 273

Westmoquette, M. S., Smith, L. J., \& Gallagher, J. S. 2008, MNRAS, 383, 864

Westmoquette, M. S., Gallagher, J. S., \& de Poitiers, L. 2010, MNRAS, 403, 1719 